\title{
1 Epigenetic scores for the circulating proteome as tools for
}

\section{2 disease prediction}

3 Danni A Gadd ${ }^{1, *}$, Robert F Hillary ${ }^{1, *}$, Daniel L McCartney ${ }^{1, *}$, Shaza B Zaghlool ${ }^{2,3^{*}}$, Anna J

4 Stevenson $^{1}$, Yipeng Cheng ${ }^{1}$, Chloe Fawns-Ritchie ${ }^{1,5}$, Cliff Nangle ${ }^{1}$, Archie Campbell ${ }^{1}$, Robin

5 Flaig $^{1}$, Sarah E Harris ${ }^{4,5}$, Rosie M Walker ${ }^{6}$, Liu Shi ${ }^{7}$, Elliot M Tucker-Drob ${ }^{8,9}$, Christian

6 Gieger $^{10,11,12,13}$, Annette Peters ${ }^{11,12,13}$, Melanie Waldenberger ${ }^{10,11,12}$, Johannes Graumann ${ }^{14,15}$,

7 Allan F McRae ${ }^{16}$, Ian J Deary ${ }^{4,5}$, David J Porteous ${ }^{1}$, Caroline Hayward ${ }^{1,17}$, Peter M

8 Visscher $^{16}$, Simon R Cox ${ }^{4,5}$, Kathryn L Evans ${ }^{1}$, Andrew M McIntosh ${ }^{1,18}$, Karsten Suhre ${ }^{2}$,

9 Riccardo E Marioni ${ }^{1, \dagger}$

$10{ }^{1}$ Centre for Genomic and Experimental Medicine, Institute of Genetics and Cancer, University of

11 Edinburgh, Edinburgh, EH4 2XU

$12{ }^{2}$ Department of Physiology and Biophysics, Weill Cornell Medicine-Qatar, Education City, PO 24144

13 Doha, Qatar

$14{ }^{3}$ Computer Engineering Department, Virginia Tech, Blacksburg, VA, USA.

$15{ }^{4}$ Lothian Birth Cohorts, University of Edinburgh, Edinburgh, EH8 9JZ

$16 \quad{ }^{5}$ Department of Psychology, University of Edinburgh, Edinburgh, EH8 9JZ

$17{ }^{6}$ Centre for Clinical Brain Sciences, Chancellor's Building, 49 Little France Crescent, Edinburgh

18 BioQuarter, Edinburgh, EH16 4SB

$19 \quad{ }^{7}$ Department of Psychiatry, University of Oxford, UK

$20{ }^{8}$ Department of Psychology, The University of Texas at Austin, United States

$21{ }^{9}$ Population Research Center, The University of Texas at Austin, United States

$22 \quad{ }^{10}$ Research Unit Molecular Epidemiology, Helmholtz Zentrum München, German Research Center

23 for Environmental Health, Neuherberg, Bavaria, Germany 
$24 \quad{ }^{11}$ Institute of Epidemiology, Helmholtz Zentrum München, German Research Center for

25 Environmental Health, Neuherberg, Bavaria, Germany

$26{ }^{12}$ German Center for Cardiovascular Research (DZHK), partner site Munich Heart Alliance, Munich,

27 Germany

$28{ }^{13}$ German Center for Diabetes Research (DZD), Neuherberg, Germany

$29{ }^{14}$ Scientific Service Group Biomolecular Mass Spectrometry, Max Planck Institute for Heart and

30 Lung Research, W.G. Kerckhoff Institute, Bad Nauheim, Germany

$31{ }^{15}$ German Centre for Cardiovascular Research (DZHK), Partner Site Rhine-Main, Max Planck

32 Institute of Heart and Lung Research, Bad Nauheim, Germany

$33 \quad{ }^{16}$ Institute for Molecular Bioscience, University of Queensland, Brisbane, QLD, Australia

$34{ }^{17}$ Medical Research Council Human Genetics Unit, Institute of Genetics and Cancer, University of

35 Edinburgh, Edinburgh, EH4 2XU

$36{ }^{18}$ Division of Psychiatry, University of Edinburgh, Royal Edinburgh Hospital, Edinburgh, EH10 5HF,

$37 \quad \mathrm{UK}$

$38 *$ These authors contributed equally

$39 \dagger$ Corresponding author: Riccardo Marioni, riccardo.marioni@ed.ac.uk

40

41

42

43

44

45

46 


\section{Abstract}

48 Protein biomarkers have been identified across many age-related morbidities. However, characterising epigenetic influences could further inform disease predictions. Here, we leverage epigenome-wide data to study links between the DNAm signatures of the circulating

51 proteome and incident diseases. Using data from four cohorts, we trained and tested epigenetic scores (EpiScores) for 953 plasma proteins, identifying 109 scores that explained between $1 \%$ and $58 \%$ of the variance in protein levels after adjusting for known protein quantitative trait loci (pQTL) genetic effects. By projecting these EpiScores into an independent sample, (Generation Scotland; $n=9,537$ ) and relating them to incident morbidities over a follow-up of 14 years, we uncovered 137 EpiScore - disease associations. These associations were largely independent of immune cell proportions, common lifestyle and health factors and biological aging. Notably, we found that our diabetes-associated EpiScores highlighted previous top biomarker associations from proteome-wide assessments of diabetes. These EpiScores for protein levels can therefore be a valuable resource for disease prediction and risk stratification.

\section{Introduction}

65 Chronic morbidities place longstanding burdens on our health as we age. Stratifying an individual's risk prior to symptom presentation is therefore critical (NHS England, 2016).

67 Though complex morbidities are partially driven by genetic factors (Fuchsberger et al., 2016;

68 Yao et al., 2018), epigenetic modifications have also been associated with disease (Lord \& 69 Cruchaga, 2014). DNA methylation (DNAm) encodes information on the epigenetic 
landscape of an individual and blood-based DNAm signatures have been found to predict allcause mortality and disease onset, providing strong evidence to suggest that methylation is an important measure of disease risk (Hillary, Stevenson, et al., 2020; Lu et al., 2019; Y. Zhang et al., 2017). DNAm can regulate gene transcription (Lea et al., 2018), and epigenetic differences can be reflected in the variability of the proteome (Hillary et al., 2019; Hillary, Trejo-Banos, et al., 2020; Zaghlool et al., 2020). Low-grade inflammation, which is thought to exacerbate many age-related morbidities, is particularly well-captured through DNAm studies of plasma protein levels (Zaghlool et al., 2020). As proteins are the primary effectors of disease, connecting the epigenome, proteome and time to disease onset may help to resolve predictive biological signatures.

Epigenetic predictors have utilised DNAm from the blood to estimate a person's 'biological age' (Lu et al., 2019), measure their exposure to lifestyle and environmental factors (McCartney, Hillary, et al., 2018; McCartney, Stevenson, Hillary, et al., 2018; Peters et al., 2021) and predict circulating levels of inflammatory proteins (A. Stevenson et al., 2020; A. J. Stevenson et al., 2021). A leading epigenetic predictor of biological aging, the GrimAge epigenetic clock incorporates methylation scores for seven proteins along with smoking and chronological age, and is associated with numerous incident disease outcomes independently of smoking (Hillary, Stevenson, et al., 2020; Lu et al., 2019). This suggests there is predictive value gained in utilising DNAm scores relevant to protein levels as intermediaries for predictions. Methylation scores also point towards the pathways that may act on health beyond the protein biomarker that they are trained on. A portfolio of methylation scores for proteins across the circulating proteome could therefore aid in the prediction of disease and offer a different, but additive signal beyond methylation or protein data alone. Generation of 
an extensive range of epigenetic scores for protein levels has not been attempted to date. The capability of specific protein scores to predict a range of morbidities has also not been tested. However, DNAm scores for Interleukin-6 and C-Reactive protein have been found to associate with a range of phenotypes independently of measured protein levels, show more stable longitudinal trajectories than repeated protein measurements, and, in some cases, outperform blood-based proteomic associations with brain morphology (Conole et al., 2020; A. J. Stevenson et al., 2021). This is likely due to DNA methylation representing the accumulation of more sustained effects over a longer period of time than protein measurements, which have often been shown to be highly variable in their levels when measured at multiple time points (Koenig et al., 2003; Liu et al., 2015; Moldoveanu et al., 2000; Shah et al., 2014). DNA methylation scores for proteins could therefore be used to alert clinicians to individuals with high-risk biological signatures, many years prior to disease onset.

Here, we report a comprehensive association study of blood-based DNAm with proteomics and disease (Figure 1). We trained epigenetic scores - referred to as EpiScores - for 953 plasma proteins (with sample size ranging from 725 - 944 individuals) and validated them using two independent cohorts with 778 and 162 participants. We regressed out known genetic pQTL effects from the protein levels prior to generating the EpiScores to preclude the signatures being driven by common SNP data that are invariant across the lifespan. We then examined whether the most robust predictors ( $n=109$ EpiScores) associated with the incidence of 12 major morbidities (Table 1), over a follow up period of up to 14 years in the Generation Scotland cohort $(n=9,537)$. We also tested for associations between EpiScore levels and COVID-19 disease outcomes. We regressed out the effects of age on protein levels 
prior to training and testing; age was also included as a covariate in disease prediction models. We controlled for common risk factors for disease and assessed the capacity of EpiScores to identify previously reported protein-disease associations.

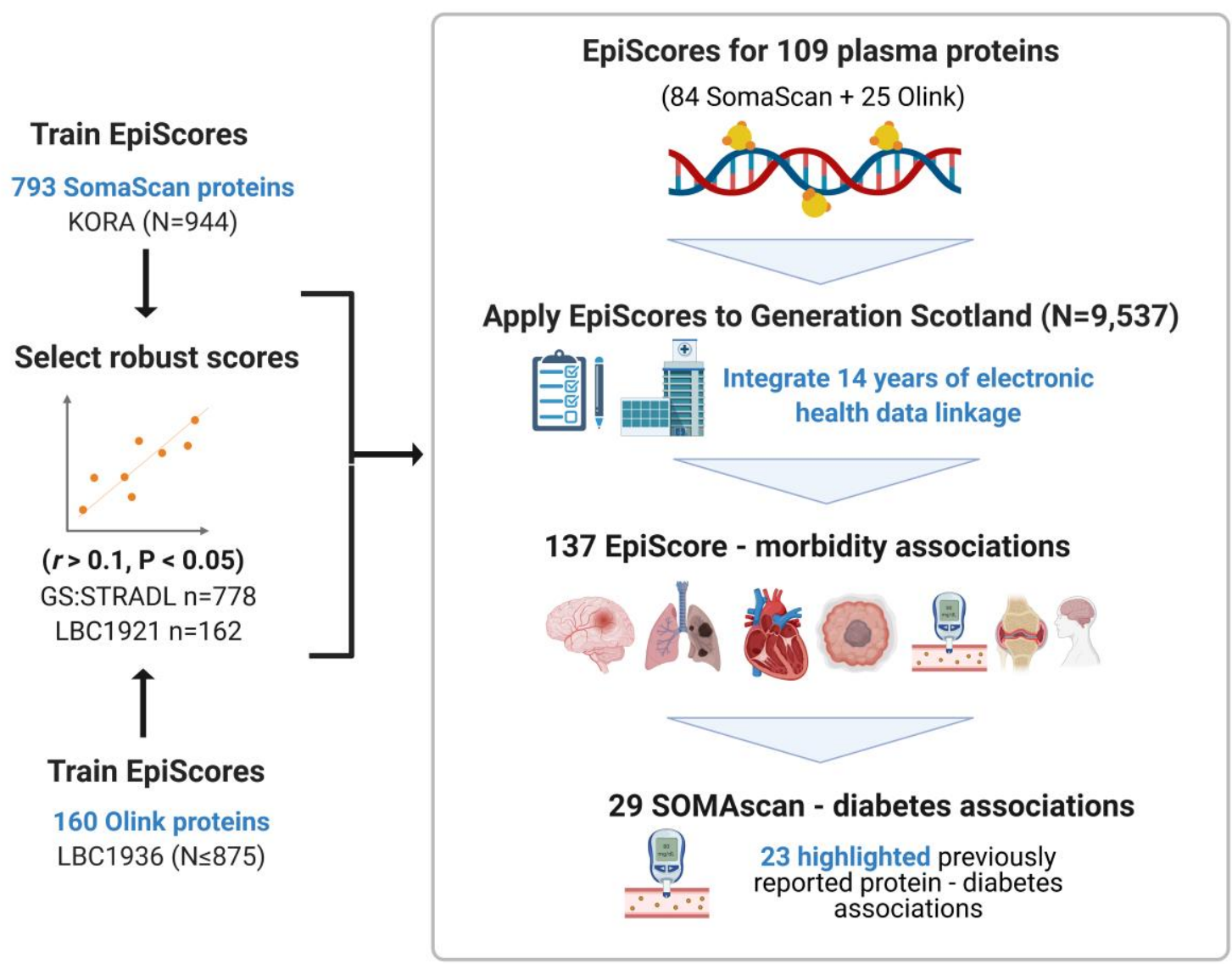

Figure 1. EpiScores for plasma proteins as tools for disease prediction study design. DNA methylation scores were trained on 953 circulating plasma protein levels in the KORA and LBC1936 cohorts. There were 109 EpiScores selected based on performance $(r>0.1, \mathrm{P}<0.05)$ in independent test sets. The selected EpiScores were projected into Generation Scotland, a cohort that has extensive data linkage to GP and hospital records. We tested whether levels of each EpiScore at baseline could predict the onset of 12 leading causes of morbidity, over a follow-up period of up to 14 years. 137 EpiScore - disease associations were identified, for 11 morbidities. We then assessed whether EpiScore associations reflected protein associations for diabetes, which is a trait that has been well-characterised using SOMAscan protein measurements. Of the 29 SOMAscan-derived EpiScore - diabetes associations, 23 reflected highlighted previously reported protein - diabetes associations. 


\begin{tabular}{lcccccc}
\hline Morbidity & $\begin{array}{c}\text { N } \\
\text { cases }\end{array}$ & $\begin{array}{c}\text { N } \\
\text { Controls }\end{array}$ & $\begin{array}{c}\text { Years to event } \\
\text { (mean, sd) }\end{array}$ & $\begin{array}{c}\text { N } \\
\text { cases }\end{array}$ & $\begin{array}{c}\text { N } \\
\text { Controls }\end{array}$ & $\begin{array}{c}\text { Years to event } \\
\text { (mean, sd) }\end{array}$ \\
\hline Rheumatoid arthritis & 65 & 9281 & $6.1(3.5)$ & 54 & 7736 & $6.4(3.3)$ \\
Alzheimer's dementia & 69 & 3764 & $8.3(2.7)$ & 52 & 3137 & $8.2(2.7)$ \\
Bowel cancer & 77 & 9398 & $6.4(3.2)$ & 65 & 7817 & $6.5(3.2)$ \\
Depression & 101 & 8306 & $3.9(3.3)$ & 80 & 6976 & $3.7(3.3)$ \\
Breast cancer & 129 & 5355 & $6(3.4)$ & 110 & 4401 & $5.9(3.4)$ \\
Lung cancer & 201 & 9265 & $5.2(3.1)$ & 172 & 7705 & $5.1(3.1)$ \\
Inflammatory bowel disease & 203 & 9083 & $5(3.5)$ & 163 & 7567 & $4.9(3.5)$ \\
Stroke & 317 & 9023 & $6.5(3.4)$ & 248 & 7546 & $6.4(3.5)$ \\
COPD & 346 & 8939 & $6.2(3.4)$ & 273 & 7459 & $6.1(3.4)$ \\
Ischaemic heart disease & 395 & 8646 & $5.8(3.3)$ & 309 & 7248 & $5.9(3.3)$ \\
Diabetes & 428 & 8756 & $5.7(3.4)$ & 322 & 7331 & $5.7(3.4)$ \\
Pain (back/neck) & 1494 & 5341 & $5.2(3.5)$ & 1221 & 4475 & $5.3(3.5)$ \\
\hline
\end{tabular}

\section{Results}

Table 1. Incident morbidities in the Generation Scotland cohort. Counts are provided for the number of cases and controls for each incident trait in the basic and fully-adjusted Cox models run in the Generation Scotland cohort $(n=9,537)$. Mean time-to-event is summarised in years for each phenotype. Alzheimer's dementia cases and controls were restricted to those older than 65 years. Breast cancer cases and controls were restricted to females. 
To generate epigenetic scores for a comprehensive set of plasma proteins, we ran elastic net penalised regression models using protein measurements from the SOMAscan (aptamerbased) and Olink (antibody-based) platforms. We used two cohorts: the German populationbased study KORA ( $\mathrm{n}=944$, mean age 59 years (SD 7.8), with 793 SOMAscan proteins) and the Scottish Lothian Birth Cohort 1936 (LBC1936) study (between 725 and 875 individuals in the training cohort, with a total of 160 Olink neurology and inflammatory panel proteins). The mean age of the LBC1936 participants at sampling was 70 (SD 0.8) for inflammatory and 73 (SD 0.7) for neurology proteins. Full demographic information is available for all cohorts in Supplementary file 1A.

Prior to running the elastic net models, we rank-based inverse normalised protein levels and adjusted for age, sex, cohort-specific variables and, where present, cis and trans pQTL effects identified from previous analyses (Hillary et al., 2019; Hillary, Trejo-Banos, et al., 2020; Suhre et al., 2017) (Methods). Of a possible 793 proteins in KORA, 84 EpiScores had Pearson $r>0.1$ and $\mathrm{P}<0.05$ when tested in an independent subset of Generation Scotland (The Stratifying Resilience and Depression Longitudinally [STRADL] study, n=778) (Supplementary file 1B). These EpiScores were selected for EpiScore-disease analyses. Of the 160 Olink proteins trained in LBC1936, there were 21 with $r>0.1$ and $\mathrm{P}<0.05$ in 174 independent test sets (STRADL, $\mathrm{n}=778$, Lothian Birth Cohort 1921: LBC1921, $\mathrm{n}=162$ ) 175 (Supplementary file 1C). Independent test set data were not available for four Olink proteins. However, they were included based on their performance $(r>0.1$ and $\mathrm{P}<0.05)$ in a holdout sample of 150 individuals who were left out of the training set. We then retrained these four predictors on the full training sample. 
179 A total of 109 EpiScores (84 SOMAscan-based and 25 Olink-based) were brought forward $(r$ $180>0.1$ and $\mathrm{P}<0.05)$ to EpiScore-disease analyses (Figure 2 and Supplementary file 1D). 181 There were five EpiScores for proteins common to both Olink and SOMAscan panels, which 182 had variable correlation strength (GZMA $r=0.71$, MMP.1 $r=0.46$, CXCL10 $r=0.35$, 183 NTRK3 $r=0.26$, and CXCL11 $r=0.09$ ). Predictor weights, positional information and cis/trans status for $\mathrm{CpG}$ sites contributing to these EpiScores are available in Supplementary file 1F. The number of $\mathrm{CpG}$ features selected for EpiScores ranged from one (Lyzozyme) to 395 (Aminoacylase-1), with a mean of 96 Supplementary file 1G). The most frequently selected CpG was the smoking-related site cg05575921 (mapping to the AHRR gene), which was included in 25 EpiScores. Counts for each CpG site are summarised in Supplementary

file 1H. This table includes the set of protein EpiScores that each $\mathrm{CpG}$ contributes to, along 190 with phenotypic annotations (traits) from the MRC-IEU EWAS catalog (MRC-IEU, 2021) for each $\mathrm{CpG}$ site having genome-wide significance $\left(\mathrm{P}<3.6 \times 10^{-8}\right.$ ) (Saffari et al., 2017). GeneSet enrichment analysis of the original proteins used to train the 109 EpiScores highlighted pathways associated with immune response and cell remodelling, adhesion and extracellular matrix function (Supplementary File 1E).

195

196

197 


\section{a}

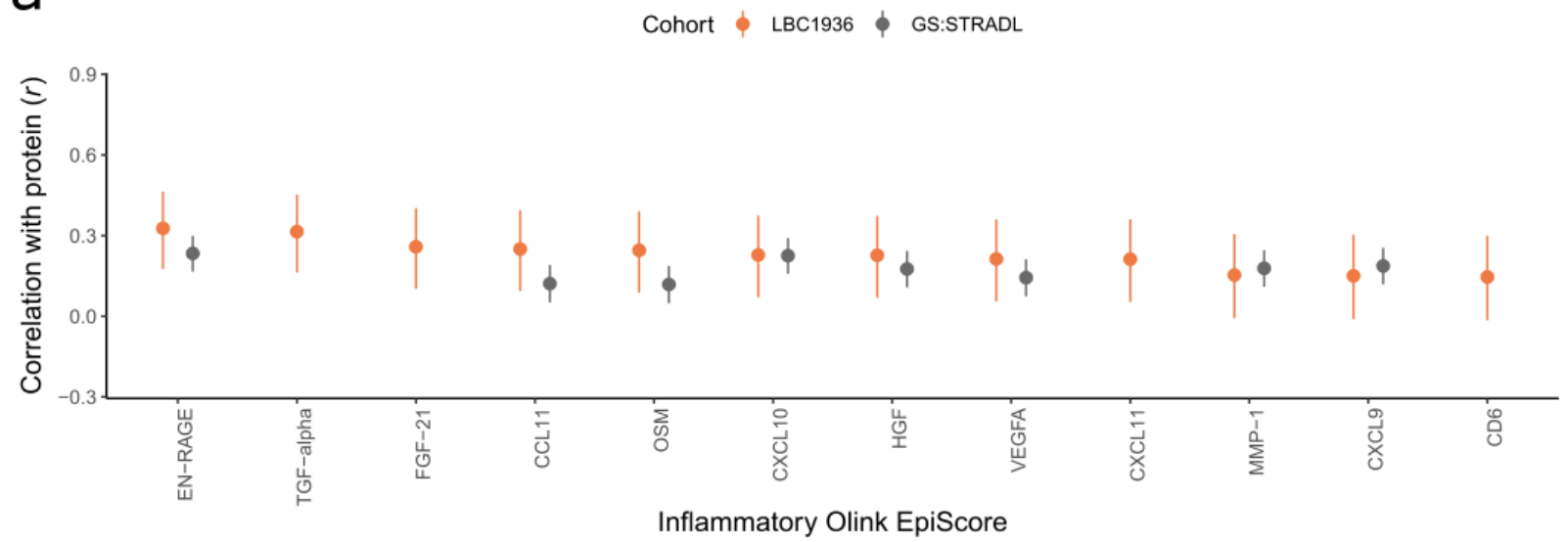

b

Cohort $\phi$ LBC1936 $\phi$ GS:STRADL $\phi$ LBC1921

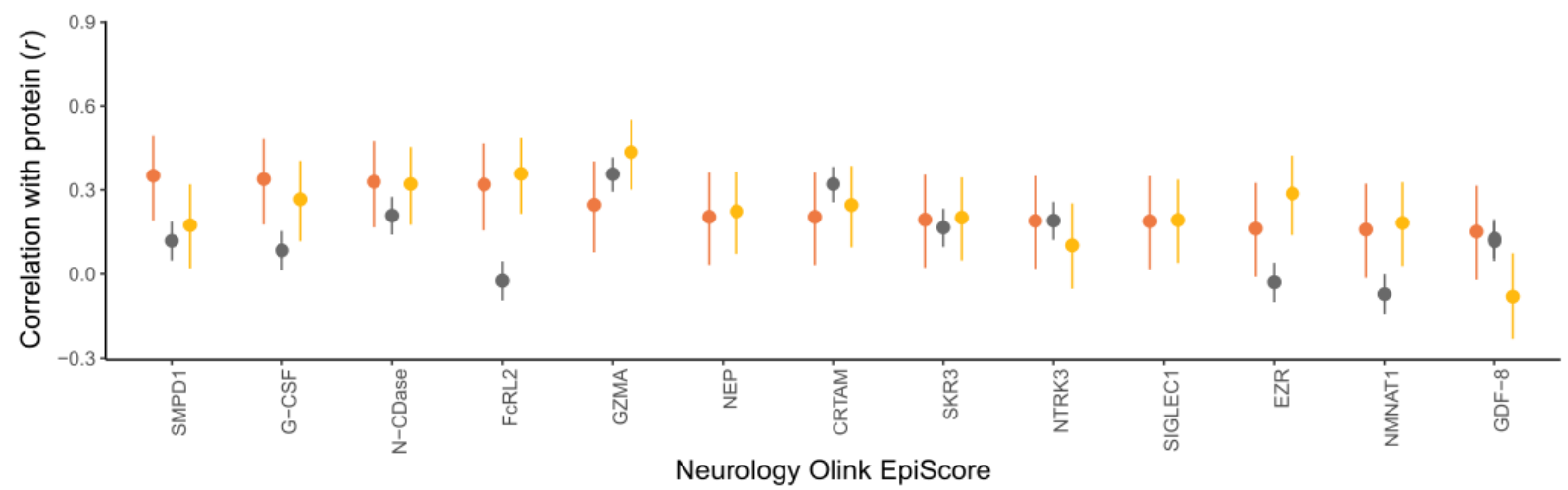

C

Cohort $\phi$ GS:STRADL

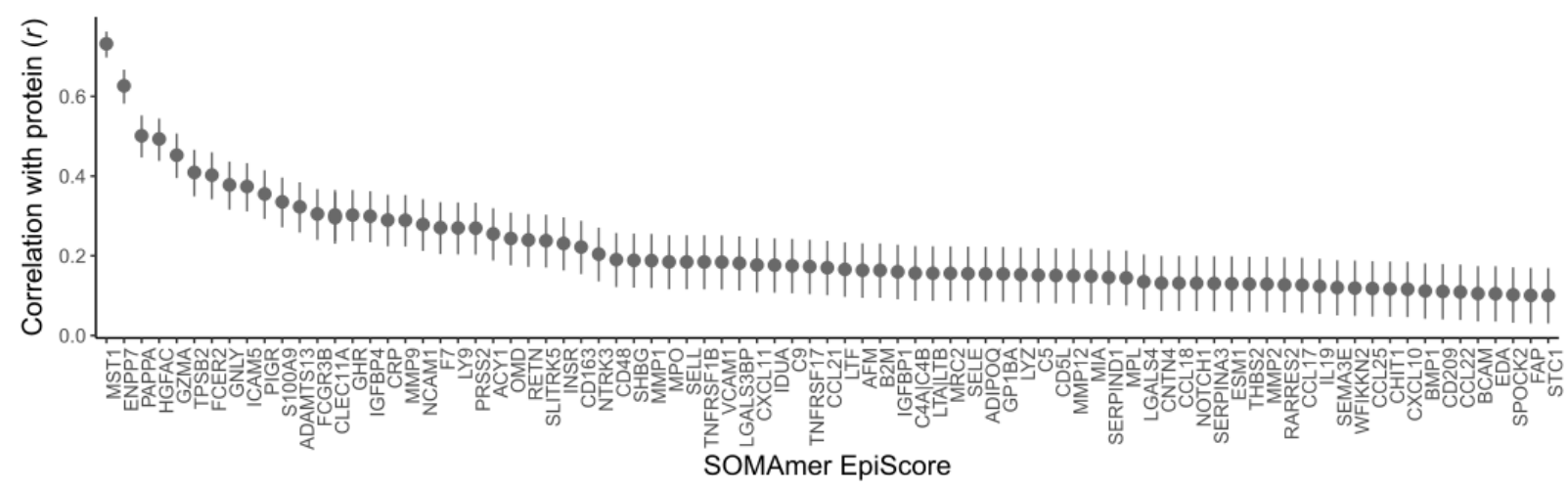

Figure 2. Test performance for the 109 selected protein EpiScores. Test set correlation coefficients for associations between protein EpiScores for (a) inflammatory Olink, (b) neurology Olink and (c) SOMAmer protein panel EpiScores and measured protein levels are plotted. Upper and lower confidence intervals are shown for each correlation. The 109 protein EpiScores shown achieved $r>0.1$ and $\mathrm{P}<0.05$ either one or both of the GS:STRADL $(\mathrm{n}=778)$ and LBC1921 $(n=162)$ test sets, wherever protein data was available for comparison. Data shown corresponds to the results included in Supplementary files 1B-C. Correlation heatmaps between the 109 EpiScore measures (Figure 2 - figure supplement 1) and between the 109 EpiScores and phenotypic traits and estimated white blood cell proportions (Figure 2 - figure supplement 2) are 
also provided, along with a summary of the most enriched functional pathways for the genes of the 109 proteins used to train EpiScores (Figure 2 - figure supplement 3).

\section{EpiScore-disease associations in Generation Scotland}

The Generation Scotland dataset contains extensive electronic health data from GP and hospital records available as well as DNA methylation data for 9,537 individuals. This makes it uniquely positioned to test whether EpiScore signals can predict disease onset. We ran nested mixed effects Cox proportional hazards models (Figure 3) to determine whether the levels of each EpiScore at baseline associated with the incidence of 12 morbidities over a maximum of 14 years of follow up. The correlation structures for the 109 EpiScore measures used for Cox modelling are presented in Figure 2 - figure supplement 1.

The Cox proportional hazard assumption dictates that hazard ratios for EpiScore - disease associations should remain constant over time. We correlated the Schoenfeld residuals from the models with time to test this. Two associations in the basic model adjusting for age and sex failed to satisfy the global assumption (across all covariates) and were excluded. There were 294 remaining EpiScore-disease associations with a False Discovery Rate (FDR)adjusted $\mathrm{P}<0.05$ in the basic model. After further adjustment for common risk factor covariates (smoking, social deprivation status, educational attainment, body mass index (BMI) and alcohol consumption), 137 of the 294 EpiScore-disease associations from the basic model had $\mathrm{P}<0.05$ in the fully-adjusted model (Supplementary files 1I-J). Eleven of the 137 fully-adjusted associations failed the Cox proportional hazards assumption for the EpiScore variable $(\mathrm{P}<0.05$ for the association between the Schoenfeld residuals and time;

Supplementary file 1K). When we restricted the time-to-event/censor period by each year of possible follow-up, there were minimal differences in the EpiScore - disease hazard ratios 
234 between follow-up periods that did not violate the assumption and those that did 235 (Supplementary file 1L). The 137 associations were therefore retained as the primary 236 results.

237 The 137 associations found in the fully-adjusted model comprised 78 unique EpiScores that were related to the incidence of 11 of the 12 morbidities studied. Diabetes and chronic obstructive pulmonary disease (COPD) had the greatest number of associations, with 33 and 41, respectively. Figure 4 presents the EpiScore-disease relationships for COPD and the remaining nine morbidities: stroke, lung cancer, ischaemic heart disease, inflammatory bowel

242 disease, rheumatoid arthritis, depression, bowel cancer, pain (back/neck) and Alzheimer's 243 dementia. There were 13 EpiScores that associated with the onset of three or more morbidities. Figure 5 presents relationships for these 13 EpiScores in the fully-adjusted Cox model results. Of note is the EpiScore for Complement 5 (C5), which associated with five outcomes: stroke, diabetes, ischaemic heart disease, rheumatoid arthritis and COPD. Of the 29 SOMAscan-derived EpiScore associations with incident diabetes, 23 replicated previously reported protein associations (Elhadad et al., 2020; Gudmundsdottir et al., 2020; Ngo et al., 2021) with incident or prevalent diabetes in one or more cohorts (Figure 6 and 
Basic model

\begin{tabular}{c}
\hline Age + Sex \\
294 \\
EpiScore-disease \\
associations \\
(FDR-adjusted \\
P $<0.05)$
\end{tabular}

Fully-adjusted

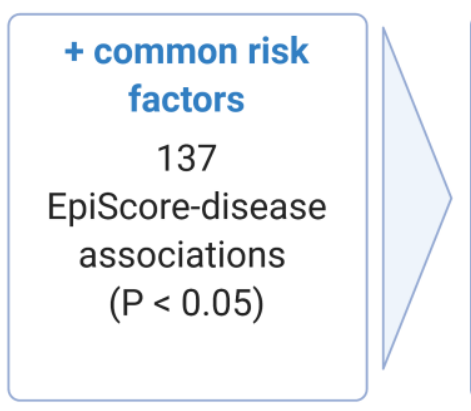

WBC-adjusted

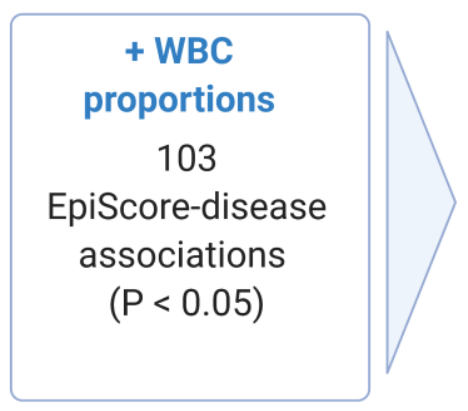

GrimAge-adjusted

+ GrimAge acceleration

81

EpiScore-disease

associations

$(P<0.05)$

257 Figure 3. Nested Cox proportional hazards assessment of protein EpiScore-disease

258 prediction. Mixed effects Cox proportional hazards analyses in Generation Scotland $(n=9,537)$

259 tested the relationships between each of the 109 selected EpiScores and the incidence of 12 leading

260 causes of morbidity (Supplementary files 1I-J). The basic model was adjusted for age and sex

261 and yielded 294 associations between EpiScores and disease diagnoses, with FDR-adjusted P <

262 0.05. In the fully-adjusted model, which included common risk factors as additional covariates

263 (smoking, deprivation, educational attainment, BMI and alcohol consumption) 137 of the basic

264 model associations remained significant with $\mathrm{P}<0.05$. In a sensitivity analysis, the addition of

265 estimated White Blood Cells (WBCs) to the fully-adjusted models led to the attenuation of 34 of

266 the 137 associations. In a further sensitivity analysis, 81 associations remained after adjustment for

267 both immune cell proportions and GrimAge acceleration. 

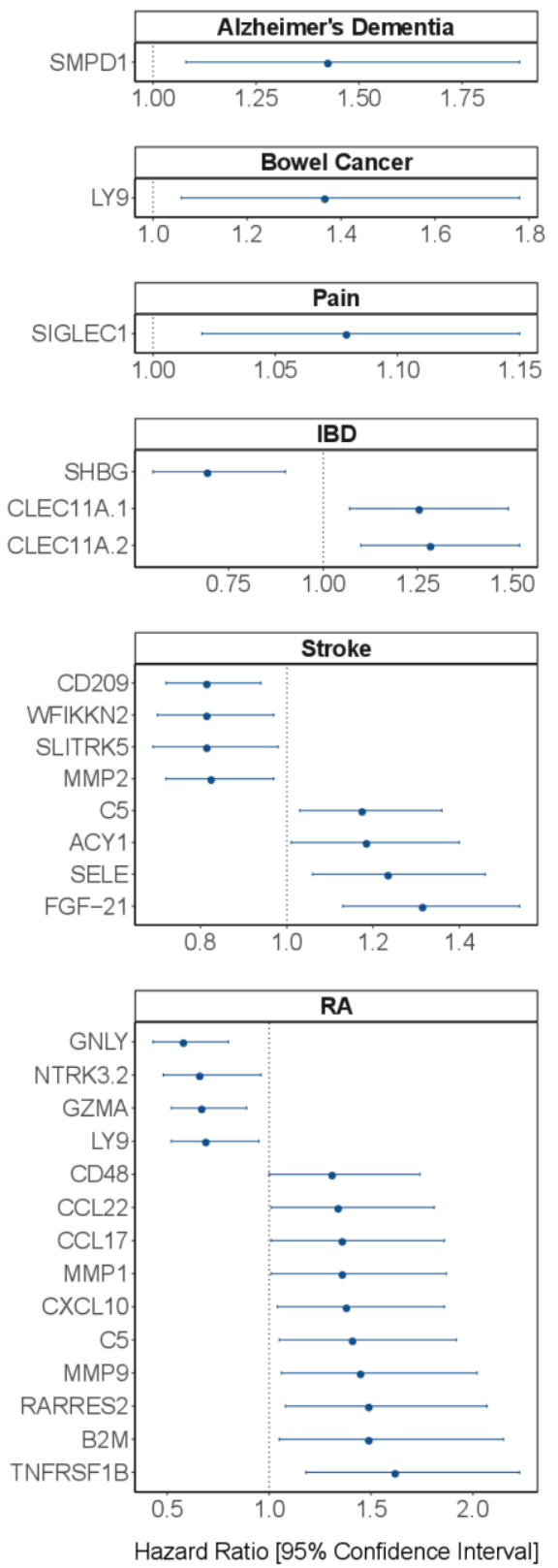
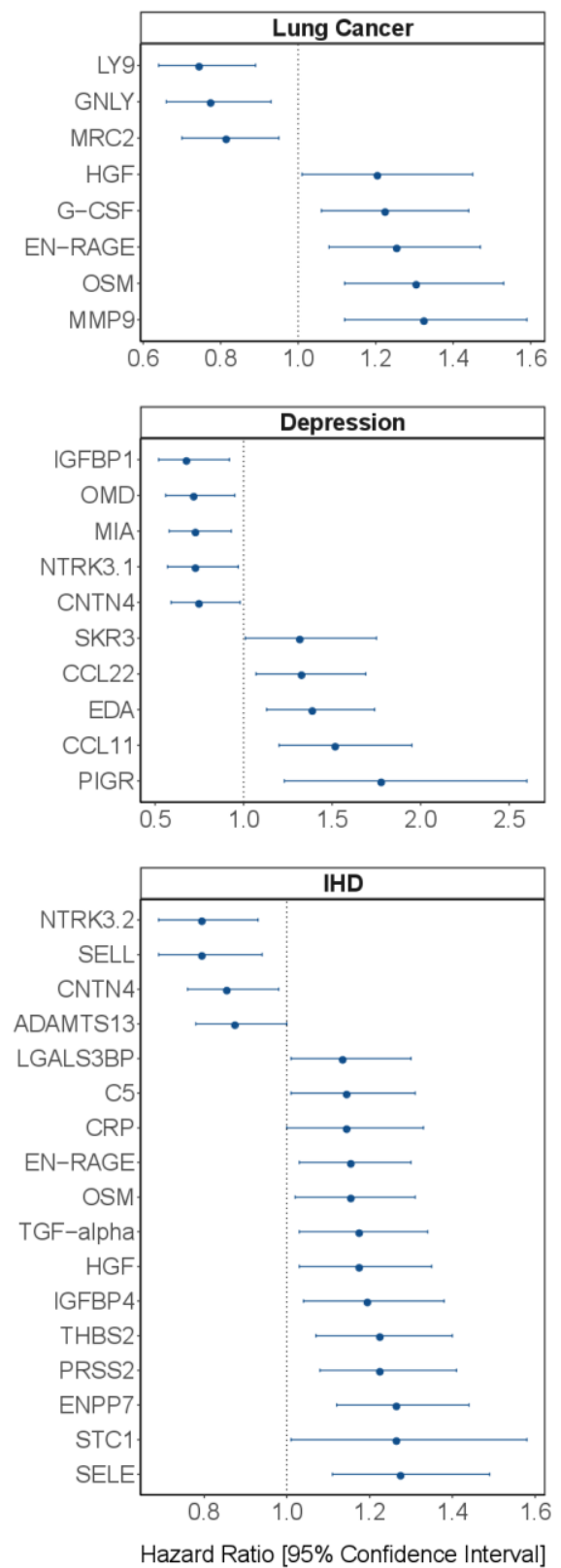

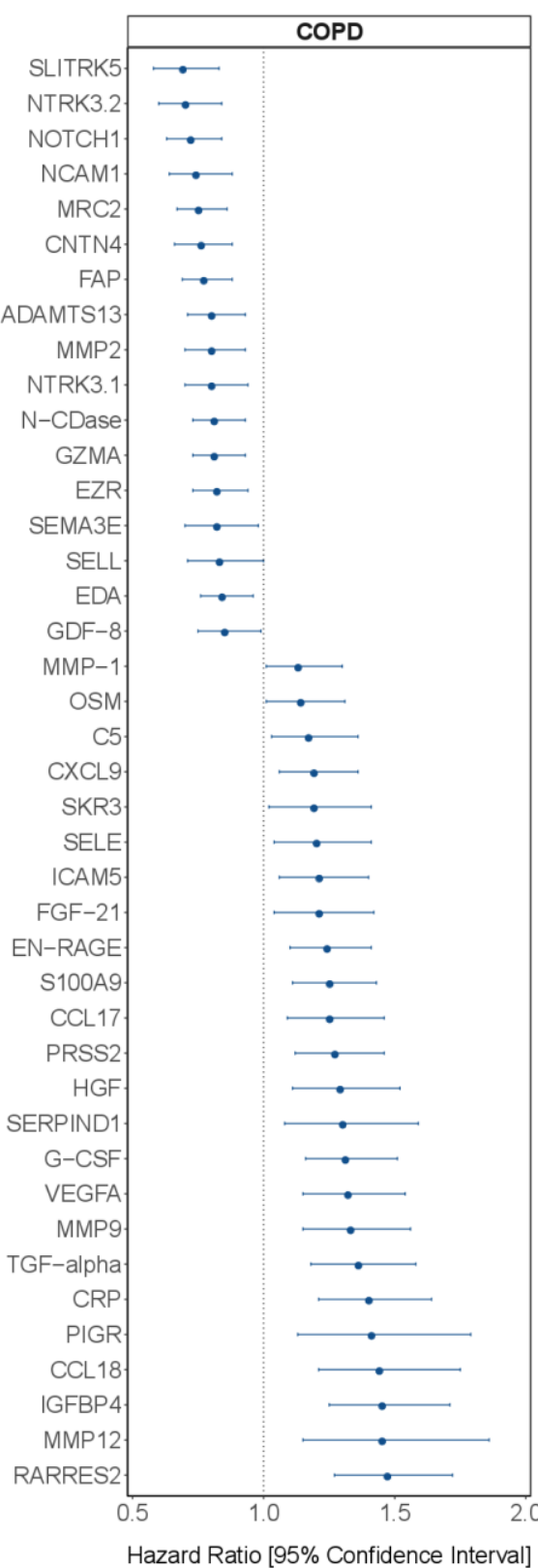

271 Figure 4. Protein EpiScore associations with incident disease. EpiScore-disease associations for

272 ten of the eleven morbidities with associations where $\mathrm{P}<0.05$ in the fully-adjusted mixed effects

273 Cox proportional hazards models in Generation Scotland $(n=9,537)$. Hazard ratios are presented

274 with confidence intervals for 104 of the 137 EpiScore - incident disease associations reported.

275 Models were adjusted for age, sex and common risk factors (smoking, BMI, alcohol consumption,

276 deprivation and educational attainment). IBD: inflammatory bowel disease. IHD: ischaemic heart

277 disease. COPD: chronic obstructive pulmonary disease. For EpiScore - diabetes associations, see

278 Figure 6. Data shown corresponds to the results included in Supplementary file 1J. 


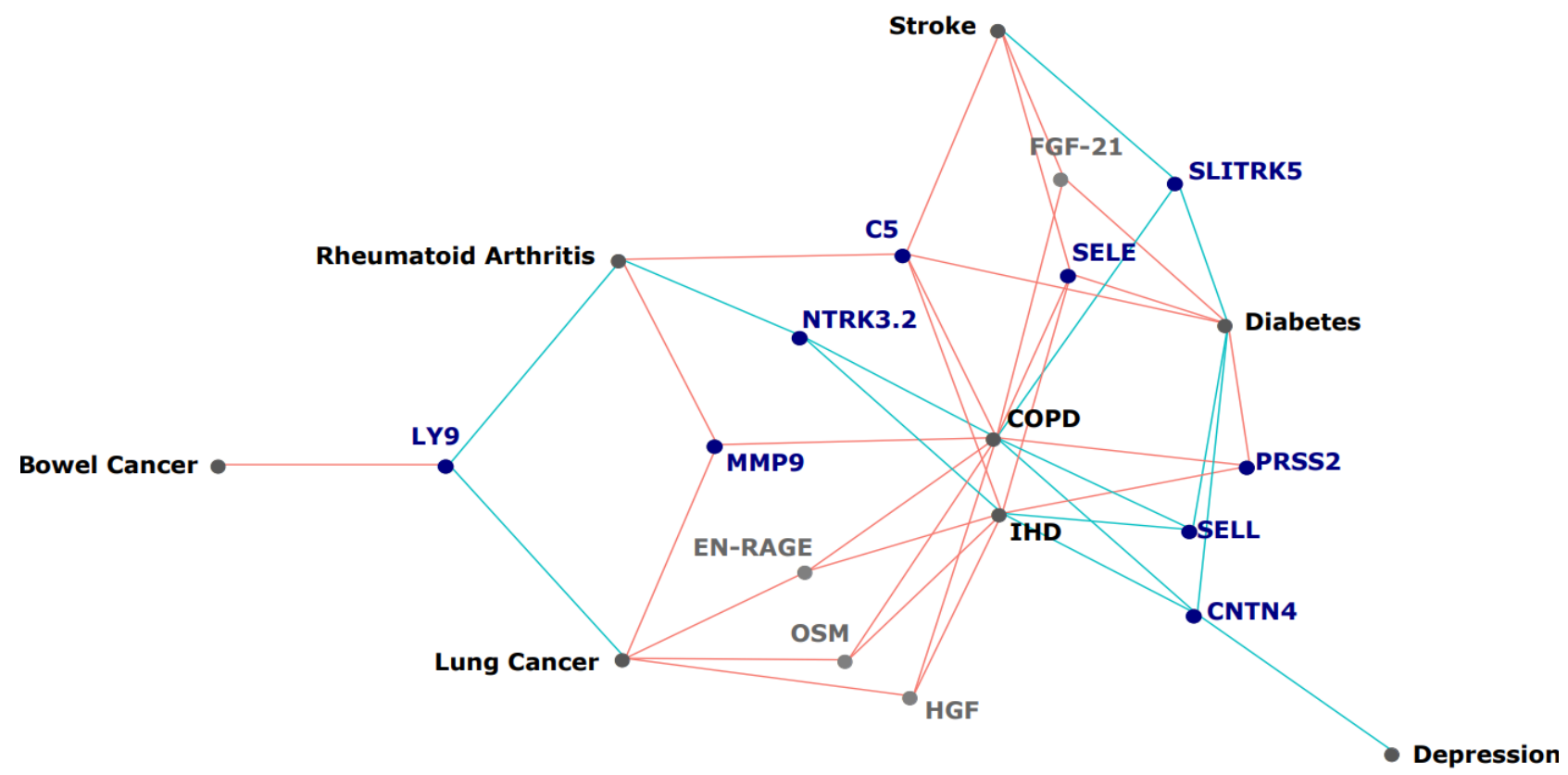

281 Figure 5. Protein EpiScores that associated with the greatest number of morbidities.

282 EpiScores with a minimum of three relationships with incident morbidities in the fully-adjusted

283 Cox models. The network includes 13 EpiScores as dark blue (SOMAscan) and grey (Olink) 284 nodes, with disease outcomes in black. EpiScore-disease associations with hazard ratios < 1 are 285 shown as blue connections, whereas hazard ratios > 1 are shown in red. COPD: chronic obstructive 286 pulmonary disease. IHD: ischaemic heart disease. Data shown corresponds to the results included 287 in Supplementary file 1J. 


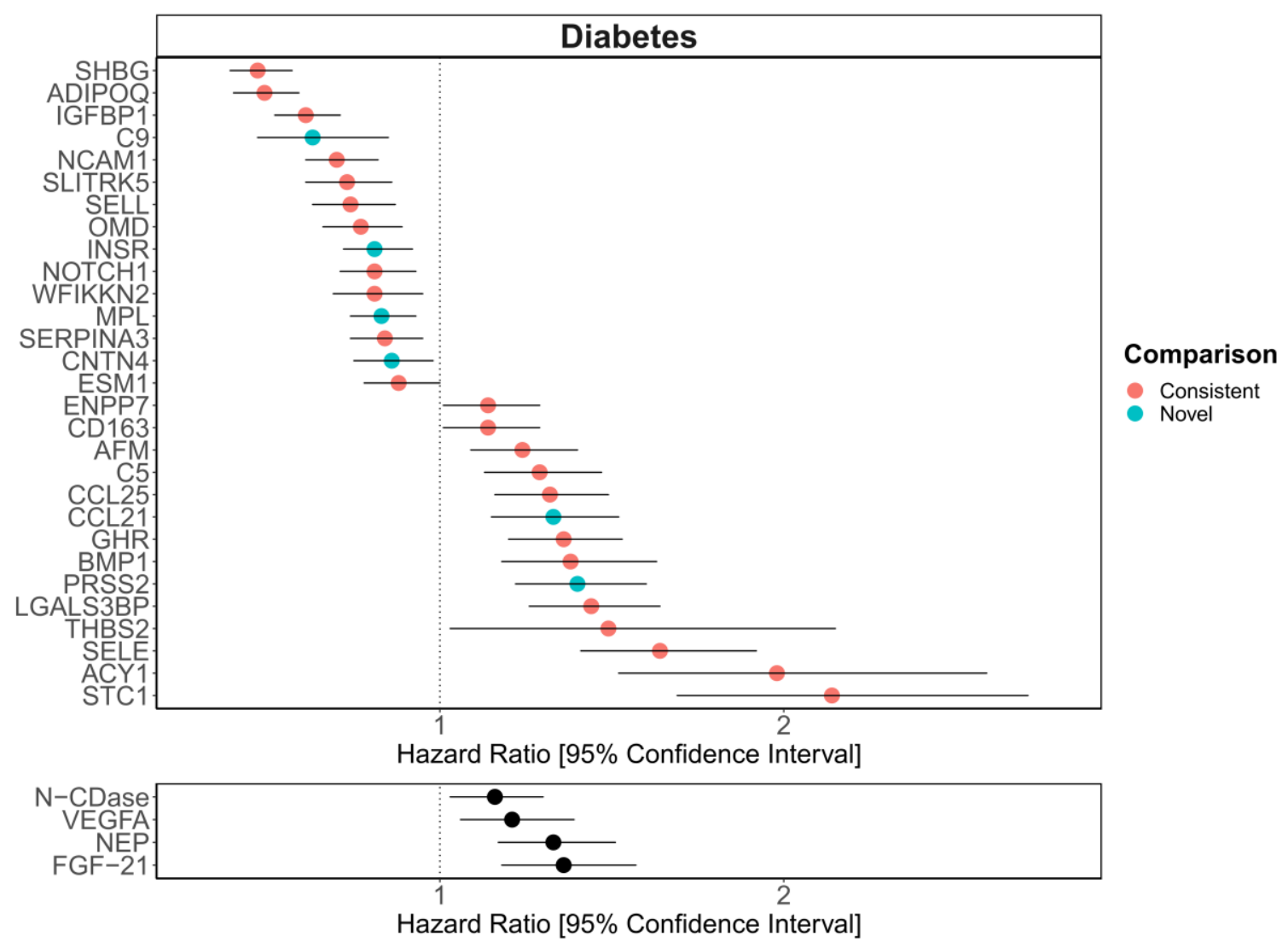

Figure 6. Replication of known protein-diabetes associations with protein EpiScores. EpiScore - incident diabetes associations in Generation Scotland $(n=9,537)$. The 29 SOMAscan (top panel) and four Olink (bottom panel) associations shown with $\mathrm{P}<0.05$ in fully-adjusted mixed effects Cox proportional hazards models. Of the 29 SOMAscan-derived EpiScores, 23 associations were consistent with protein - diabetes associations (pink) in one or more of the comparison studies that used SOMAscan protein levels. Six associations were novel (blue). Data shown corresponds to the results included in Supplementary files 1J and 1M .

\section{Immune cell and GrimAge sensitivity analyses}

Correlations of the 109 EpiScores with covariates suggested interlinked relationships with both immune cells and GrimAge acceleration (Figure 2 - figure supplement 2). These covariates were therefore added incrementally to the fully-adjusted Cox models (Figure 3). There were 103 associations that remained statistically significant (FDR P $<0.05$ in the basic model and $\mathrm{P}<0.05$ in the fully-adjusted model) after adjustment for immune cell 
proportions, of which 81 remained significant when GrimAge acceleration scores were added

317 to this model (Supplementary file 1J). In a further sensitivity analysis, relationships between both estimated White Blood Cell (WBC) proportions and GrimAge acceleration scores with incident diseases were assessed in the Cox model structure independently of EpiScores. Of the 60 possible relationships between WBC measures and the morbidities assessed, four were statistically significant (FDR-adjusted $\mathrm{P}<0.05)$ in the basic model and remained significant with $\mathrm{P}<0.05$ in the fully-adjusted model (Supplementary file $1 \mathbf{N}$ ). A higher proportion of Natural Killer cells was linked to decreased risk of incident COPD, rheumatoid arthritis, diabetes and pain (back/neck). The GrimAge acceleration composite score was associated with COPD, IHD, Diabetes and Pain (back/neck) in the fully-adjusted models $(\mathrm{P}<0.05)$ (Supplementary file 10). The magnitude of the GrimAge effect sizes were comparable to the EpiScore findings.

Relationship between EpiScores and subsequent COVID-19

Two previous studies utilising pilot proteomic data from the Generation Scotland cohort $(\mathrm{N}=199)$ as part of wider analyses have implicated several proteins that we have trained EpiScores for as associated with COVID-19 outcomes (Demichev et al., 2021; Messner et al., 2020). These included proteins such as CRP, C9, SELL and SHBG, all of which were associated with one or more incident diseases in this study. Two subsets ( $\mathrm{N}=268$ and $\mathrm{N}=173$ ) of the Generation Scotland sample who contracted COVID-19 were therefore used to test the hypothesis that EpiScores would associate with COVID-19 outcomes. No significant associations were identified that delineated differences between the risk of developing longcovid (duration $>4$ weeks) or hospitalisation from COVID-19 (associations that had $\mathrm{P}<0.05$ did not withstand Bonferroni adjustment for multiple testing) (Supplementary file 1P). 


\section{Discussion}

344 Here, we report a comprehensive DNA methylation scoring study of 953 circulating proteins.

345 We define 109 robust EpiScores for plasma protein levels that are independent of known 346 pQTL effects. By projecting these EpiScores into a large cohort with extant data linkage, we 347 show that 78 EpiScores associate with the incidence of 11 leading causes of morbidity (137 348 EpiScore - disease associations in total), but do not associate with COVID-19 outcomes. 349 Finally, we show that EpiScore - diabetes associations highlight previously measured protein 350 - diabetes relationships. The bulk of EpiScore-disease associations are independent of common lifestyle and health factors, differences in immune cell composition and GrimAge acceleration. EpiScores therefore provide methylation-proteomic signatures for disease prediction and risk stratification.

The consistency between our EpiScore - diabetes associations and previously identified protein - diabetes relationships (Elhadad et al., 2020; Gudmundsdottir et al., 2020; Ngo et al., 2021) suggests that epigenetic scores identify disease-relevant biological signals. In addition to the comprehensive lookup of SOMAscan proteins with diabetes, several of the markers we identified for COPD and IHD also reflect previous associations with measured proteins (Ganz et al., 2016; Serban et al., 2021). Thethree studies used for the diabetes comparison represent the largest candidate protein characterisations of diabetes to date and the top markers 362 identified included aminoacylase-1 (ACY-1), sex hormone binding globulin (SHBG), growth 
hormone receptor (GHR) and Insulin-like growth factor-binding protein 2 (IGFBP-2)

364 (Elhadad et al., 2020; Gudmundsdottir et al., 2020; Ngo et al., 2021) Our EpiScores for these top markers are also associated with diabetes, in addition to EpiScores for several other protein markers reported in these studies. A growing body of evidence suggests that type 2 diabetes is mediated by genetic and epigenetic regulators (Kwak \& Park, 2016) and proteins such as ACY-1 and GHR are thought to influence a range of diabetes-associated metabolic mechanisms (Kim \& Park, 2017; Pérez-Pérez et al., 2012). Proteins that we identify through EpiScore associations, such as NTR domain-containing protein 2 (WFIKKN2), have also been causally implicated in diabetes onset through Mendelian randomisation analysis (Ngo et al., 2021). In the case of diabetes, EpiScores may therefore be used as disease-relevant risk biomarkers, many years prior to onset. Validation should be tested when sufficient data become available for the remaining morbidities.

With modest test set performances (for example, SHBG $r=0.18$ and ACY-1 $r=0.25$ ), it is perhaps surprising that such strong synergy is observed between EpiScores for proteins that associated with diabetes and the trends seen with measured proteins. Nonetheless, DNA methylation scores for CRP and IL6 have previously been shown to perform modestly in test sets $(r \sim 0.2$, equivalent to $\sim 4 \%$ explained variance in protein level), but augment and often outperform the measured protein related to a range of phenotypes (A. Stevenson et al., 2020; A. J. Stevenson et al., 2021). Compared to scores utilising DNAm for the prediction of singular diseases, our EpiScores enable the granular study of individual protein predictor signatures with clinical outcomes. For example, levels of the acid sphingomyelinase (ASM) EpiScore predicted onset of Alzheimer's dementia, several years prior to diagnosis. ASM (encoded by SMPD1) has been discussed as a therapeutic candidate for Alzheimer's disease 
387 (Cataldo et al., 2004; Kamil et al., 2016; Lee et al., 2014; Park et al., 2020) and has been shown to disrupt autophagic protein degradation and associate with accumulation of amyloidbeta in murine models of Alzheimer's pathology (Lee et al., 2014; Park et al., 2020).

Our large-scale assessment of EpiScores provides a platform for future studies, as composite predictors for traits may be created using our EpiScore database. These should be tested in incident disease predictions when sufficient case data are available. Our results indicated that the set of 109 EpiScores are likely to be heavily enriched for inflammatory, complement system, innate immune system pathways, in addition to extracellular matrix, cell remodelling and cell adhesion pathways. This reinforces previous work linking chronic inflammation and the epigenome (Zaghlool et al., 2020). It also suggests that EpiScores could be useful in the prediction of morbidities that are characterised by pro-inflammatory states. An example of this is the EpiScore for Complement Component 5 (C5), which was associated with the onset of five morbidities, the highest number for any EpiScore (Figure 5). The EpiScore for C5 is likely to reflect the biological pathways occurring in individuals with heightened complement cascade activity and could be utilised to alert clinicians to individuals at high risk of multimorbidity. Elevated levels of C5 peptides have been associated with severe inflammatory, autoimmune and neurodegenerative states (Ma et al., 2019; Mantovani et al., 2014; Morgan \& Harris, 2015) and a range of C5-targetting therapeutic approaches are in development (Alawieh et al., 2018; Brandolini et al., 2019; Hawksworth et al., 2017; Hernandez et al., 2017; Morgan \& Harris, 2015; Ort et al., 2020).

Though EpiScores such as C5 - which occupy central hubs in the disease-prediction framework - may provide evidence of early methylation signatures common to the onset of multiple diseases, we did not observe associations between EpiScores and COVID-19 
hospitalisation or long-COVID status. This is surprising, given that many of the morbidities that EpiScores predicted are also known risk factors for increased risk of death due to COVID-19 (Williamson et al., 2020). Many of the EpiScores were also found to be associated with incident diseases were trained on the levels of proteins that have been implicated in two previous studies that identified markers for COVID-19 severity and progression, using a pilot sample $(\mathrm{N}=199)$ from the Generation Scotland cohort at baseline

417 (Demichev et al., 2021; Messner et al., 2020). COVID-19 likely has multiple intersecting risk factors that impact severity and recovery and the lack of associations we observe is likely to be in part due to limited COVID-19 cases available in Generation Scotland. Additionally, there is a large lag time between baseline biological measurement and COVID-19 in our analyses, whereas the two studies that found protein marker associations integrated protein measurements longitudinally and from samples extracted during COVID-19 progression. With increased power available through continued data linkage, EpiScore relationships with COVID-19 outcomes may be observed in future work.

This study has several limitations. First, we demonstrate that EpiScores carry disease-relevant signals that may be clinically meaningful to delineate early disease risk when comparing relative differences within a cohort. However, projecting a new individual onto a reference set is complicated due to absolute differences in methylation quantification resulting from batch and processing effects. Second, future studies should assess paired protein and EpiScore contributions to traits, as inference from EpiScores alone, while useful for disease risk stratification, is not sufficient to determine mechanisms. This may also highlight 433 EpiScores that outperform the measured protein equivalent in disease. Third, the epitope nature of the protein measurement in the SOMAscan panel may incur probe cross-reactivity 
and non-specific binding; there may also be differences in how certain proteins are measured across panels (Pietzner et al., 2020; Sun et al., 2018). Comparisons of both protein measurement technologies on the same samples would help to explore this in more detail. Fourth, there may be pQTLs with small effect sizes that were not regressed from the proteins prior to generating the EpiScores. Fifth, while training and testing was performed across multiple cohorts, it is likely that further development of EpiScores in larger proteomic samples with diverse ancestries will improve power to generate robust scores. Upper bounds for DNAm prediction of complex traits, such as proteins, can be estimated by variance components analyses (Hillary, Trejo-Banos, et al., 2020; Trejo Banos et al., 2020; F. Zhang et al., 2019). Finally, associations present between EpiScore measures and disease incidence may have been influenced by external factors such as prescription medications for comorbid conditions and comorbid disease prevalence.

We have shown that EpiScores for circulating protein levels predict the incidence of multiple diseases, up to 14 years prior to diagnosis. Our findings suggest that DNA methylation phenotyping approaches and data linkage to electronic health records in large, populationbased studies have the potential to (1) Capture inter-individual variability in protein levels; (2) predict incident disease risk many years prior to morbidity onset; and (3) highlight disease-relevant biological signals for further exploration. The EpiScore weights are publicly available, enabling any cohort with Illumina DNAm data to generate them and to relate them to various outcomes. Given the increasingly widespread assessment of DNAm in cohort studies (McCartney et al., 2020; Min et al., 2020), EpiScores offer an affordable and consistent (i.e. array-based) way to utilise these signatures. This information is likely to be important in risk stratification and prevention of age-related morbidities. 
460 Materials and Methods

461

462

The KORA sample population

463

464 The KORA F4 study includes 3,080 participants who reside in Southern Germany. 465 Individuals were between 32 and 81 years of age when recruited to the study from 2006 and 466 2008. In the current study, there were 944 individuals with methylation, proteomics and 467 genetic data available. The Infinium HumanMethylation450 BeadChip platform was used to generate DNA methylation data for these individuals. The Affymetrix Axiom array was used to generate genotyping data and the SOMAscan platform was used to generate proteomic measurements in the sample.

DNA methylation in KORA

473

474 Methylation data were generated for 1,814 individuals(Petersen et al., 2014); 944 also had 475 protein and genotype measurements available. During preprocessing, 65 SNP probes were 476 excluded and background correction was performed in minfi (Aryee et al., 2014). Samples 477 with a detection rate of less than $95 \%$ were excluded. Next, the minfi R package was used to 478 perform normalization on the intensity methylation measures (Aryee et al., 2014), with a method consistent with the Lumi:QN +BMIQ pipeline. After excluding non-cg sites and 
480

481

482

483

484

485

486

487

488

489

490

491

492

493

494

495

496

497

498

499

500

501

502

CpGs on sex chromosomes or with fewer than 100 measures available, 470,837 CpGs were available for analyses.

\section{Proteomics in KORA}

The SOMAscan platform (V3.2) (Gold et al., 2010) was used to quantify protein levels in undepleted plasma for 1129 SOMAmer probes (Suhre et al., 2017). Of the 1,000 samples provided for analysis, two samples were excluded due to errors in bio-bank sampling and one based on quality control measures. Of the 997 samples available, there were 944 individuals with methylation and genotypic data. Of the 1,129 probes available, five failed the QC, leaving a total of 1,124 probes for the subsequent analysis. Protein measurements were transformed by rank-based inverse normalisation and regressed onto age, sex, known pQTLs and 20 genetic principal components of ancestry derived from the Affymetrix Axiom Array to control for population structure. pQTLs for each protein were taken from a previous GWAS in the sample (Suhre et al., 2017).

\section{The LBC1936 and LBC1921 sample populations}

The Lothian Birth Cohorts of 1921 (LBC1921; N = 550) and 1936 (LBC1936; N = 1091) are longitudinal studies of aging in individuals who reside in Scotland (Deary et al., 2012; Taylor et al., 2018). Participants completed an intelligence test at age 11 years and were recruited for these cohorts at mean ages of 79 (LBC1921) and 70 (LBC1936). They have been followed up triennially for a series of cognitive, clinical, physical and social data, along with 
503

504

505

506

507

508

509

510

511

512

513

514

515

516

517

518

519

520

521

522 Plasma samples were analysed using either the Olink® neurology 92-plex or the Olink® 523

524

525

blood donations that have been used for genetic, epigenetic, and proteomic measurement.

DNAm, proteomic (Olink® platform) and genetic data for up to 875 individuals from Waves 1 and 2 of the LBC1936 (at mean ages 70 and 73 years) and 162 individuals at Wave 3 of the LBC1921 (at mean age 87 years).

\section{DNAm in LBC1936 and LBC1921}

DNA from whole blood was assessed using the Illumina $450 \mathrm{~K}$ methylation array. Details of quality control have been described elsewhere (Shah et al., 2014; Q. Zhang et al., 2018). Raw intensity data were background-corrected and normalised using internal controls. Manual inspection resulted in the removal of low quality samples that presented issues related to bisulphite conversion, staining signal, inadequate hybridisation or nucleotide extension. Probes with low detection rate $<95 \%$ at $\mathrm{P}<0.01$ and samples with low call rates $(<450,000$ probes detected at $\mathrm{P}<0.01)$ were removed. Samples were also removed if they had a poor match between genotype and SNP control probes, or incorrect DNA methylation-predicted sex.

\section{Proteomics in LBC1936 and LBC1921} inflammation 92-plex proximity extension assays (Olink® Bioscience, Uppsala Sweden). One inflammatory panel protein (BDNF) failed quality control and was removed. A further 21 proteins were removed, as over $40 \%$ of samples fell below the lowest limit of detection. 
526 Two neurology proteins, MAPT and HAGH, were excluded due to $>40 \%$ of observations

527 being below the lower limit of detection. This resulted in 90 neurology (LBC1936 Wave 2)

528 and 70 inflammatory (LBC1936 Wave 1) proteins in LBC1936 and 92 neurology proteins

529 available in LBC1921. Protein levels were rank-based inverse normalised and regressed onto

530 age, sex, four genetic components of ancestry derived from multidimensional scaling of the

531 Illumina 610-Quadv1 genotype array and Olink® array plate. In LBC1936, pQTLs were 532 adjusted for, through reference to GWAS in the samples (Hillary et al., 2019; Hillary, Trejo533 Banos, et al., 2020).

537 Generation Scotland: the Scottish Family Health Study (GS) is a large, family-structured, population-based cohort study of $>24,000$ individuals from Scotland (mean age 48 years)(Smith et al., 2013). Recruitment took place between 2006 and 2011 with a clinical visit where detailed health, cognitive, and lifestyle information was collected along with biological samples (blood, urine, saliva). In GS, there were 9,537 individuals with DNAm and phenotypic information available. The Stratifying Resilience and Depression

543 Longitudinally (STRADL) cohort is a subset of 1,188 individuals from the GS cohort who 544 undertook additional assessments approximately five years after the study baseline (Navrady 545 et al., 2018). 
549 In the GS cohort, blood-based DNA methylation was generated in two sets using the Illumina 550 EPIC array. Set 1 comprised 5,190 related individuals whereas Set 2 comprised 4,583

551 individuals, unrelated to each other and to those in Set 1. During quality control, probes were 552 removed based on visual outlier inspection, bead count $<3$ in over $5 \%$ of samples and 553 samples with detection P value below adequate thresholds (McCartney, Stevenson, Walker, et 554 al., 2018; Seeboth et al., 2020). Samples were removed based on sex mismatches, low 555 detection $\mathrm{P}$ values for CpGs and saliva samples and genetic outliers (Amador et al., 2015). 556 The quality-controlled dataset comprised 9,537 individuals $\left(\mathrm{n}_{\text {Set } 1}=5,087, \mathrm{n}_{\text {Set } 2}=4,450\right)$. The 557 same steps were also applied to process DNAm in STRADL.

\section{Proteomics in STRADL}

Measurements for 4,235 proteins in 1,065 individuals from the STRADL cohort were recorded using the SOMAscan ${ }^{\circledR}$ technology. 793 epitopes matched between the KORA and STRADL cohorts and were included for training in KORA and testing in STRADL. There were 778 individuals with proteomics data and DNAm data in STRADL. Protein measurements were transformed by rank-based inverse normalisation and regressed onto age, sex and 20 genetic principal components (derived from multidimensional scaling of genotype data from the Illumina 610-Quadv1 array). 
571 Over $98 \%$ of GS participants consented to allow access to electronic health records via data 572 linkage to GP records ( $\operatorname{Read} 2$ codes) and hospital records (ICD codes). Data are available 573 prospectively from the time of blood draw, yielding up to 14 years of linkage. We considered 574 incident data for 12 morbidities. Ten of the diseases are listed by the world health 575 organisation (WHO) as leading causes of either morbidity or mortality (Hay et al., 2017;

576 World Health Organization, 2018). Inflammatory bowel disease (IBD) (Kassam et al., 2014) 577 and rheumatoid arthritis (RA) (James et al., 2018) are also included as traits as they have 578 been reported as leading causes of disability and morbidity and the global burdens of these 579 diseases are rising (Alatab et al., 2020; Safiri et al., 2019). Prevalent cases (ascertained via 580 retrospective ICD and Read 2 codes or self-report from a baseline questionnaire) were excluded. For inflammatory bowel disease (IBD) prevalent cases were excluded based on data linkage alone. Included and excluded terms can be found in Supplementary file 1Q1B1. Alzheimer's dementia was limited to cases/controls with age of event/censoring $\geq 65$ years. Breast cancer was restricted to females only. Recurrent, major and moderate episodes of depression were included in depression. Diabetes was comprised of type 2 diabetes and more general diabetes codes such as diabetic retinopathy and diabetes mellitus with renal manifestation. Type 1 and juvenile diabetes cases were excluded.

Elastic net protein EpiScores

590

Penalised regression models were generated for 160 proteins in LBC1936 and 793 proteins in 592 KORA using Glmnet (Version 4.0-2) (Friedman et al., 2010) in R (Version 4.0) (R, 2020). 593 Protein levels were the outcome and there were $428,489 \mathrm{CpG}$ features per model in the 594 LBC1936 training and 397,630 in the KORA training. An elastic net penalty was specified 
$($ alpha $=0.5)$ and cross validation was applied. DNAm and protein measurements were scaled to have a mean of zero and variance of one.

In the KORA analyses, 10-fold cross validation was applied and EpiScores were tested in STRADL $(n=778)$. Of 480 EpiScores that generated $\geq 1$ CpG features, 84 had Pearson $r>0.1$ and $\mathrm{P}<0.05$ in STRADL. As test set comparisons were not available for every protein in the LBC1936 analyses, a holdout sample was defined, with two folds set aside as test data and 10 -fold cross validation carried out on the remaining data $\left(n_{\text {train }}=576, n_{\text {test }}=130\right.$ for neurology and $n_{\text {train }}=725, n_{\text {test }}=150$ for inflammatory proteins). We retained 36 EpiScores with Pearson $r$ $>0.1$ and $\mathrm{P}<0.05$. New predictors for these 36 proteins were then generated using 12 -fold cross validation and tested externally in STRADL $(n=778)$ and LBC1921 $(n=162$, for the neurology panel). 21 EpiScores had $r>0.1$ and $\mathrm{P}<0.05$ in at least one of the external test sets. Four EpiScores did not have external comparisons and were included based on holdout performance.

Functional annotations for each of the proteins used to train the finalised set of 109 EpiScores were sourced from the STRING database (Jensen et al., 2009). GeneSet enrichment analysis against protein-coding genes was performed using the FUMA database, to quantify which canonical pathways were most commonly implicated across the 109 genes corresponding to the proteins used to train the 109 EpiScores (Watanabe et al., 2017). The background geneset was specified as protein coding genes and a threshold of FDR $\mathrm{P}<0.05$ was applied for enrichment status, with the minimum overlapping genes with gene-sets set to greater than or equal to two.

The 109 selected EPiScores were then applied to Generation Scotland $(n=9,537)$. DNAm at each CpG site was scaled to have a mean of zero and variance of one, with scaling performed separately for GS Sets. 
622 Mixed effects Cox proportional hazards regression models adjusting for age, sex, and methylation set were used to assess the relationship between 109 EpiScores and 12 morbidities in Generation Scotland. Models were run using coxme (Therneau, 2020b) (Version 2.2-16) with a kinship matrix accounting for relatedness in Set 1. Cases included those diagnosed after baseline who had died, in addition to those who received a diagnosis and remained alive. Controls were censored if disease free at time of death, or at the end of the follow-up period. EpiScore levels were rank-base inverse normalised. Fully-adjusted models included: the following additional covariates measured at baseline: alcohol consumption (units consumed in the previous week); deprivation (assessed by the Scottish Index of Multiple Deprivation (GovScot, 2016)); body mass index $\left(\mathrm{kg} / \mathrm{m}^{2}\right)$; educational attainment (an 11-category ordinal variable) and a DNAm-based score for smoking status (Bollepalli et al., 2019). A false discovery rate multiple testing correction $\mathrm{P}<0.05$ was applied to the 1306 EpiScore-disease associations (109 EpiScores by 12 incident disease traits, with 2 associations excluded for failing the global proportional hazards assumption). Proportional hazards assumptions were checked through Schoenfeld residuals (global test and a test for the protein-EpiScore variable) using the coxph and cox.zph functions from the survival package (Therneau, 2020a) (Version 3.2-7). For each association failing to meet the assumption (Schoenfeld residuals $\mathrm{P}<0.05$ ), a sensitivity analysis was run across yearly follow-up intervals.

641 Fully-adjusted Cox proportional hazards models were run with Houseman-estimated White Blood Cell (WBC) proportions as covariates (Houseman et al., 2012). A further sensitivity 
analyses added GrimAge acceleration ( $\mathrm{Lu}$ et al., 2019) as an additional covariate. Basic and 644 fully-adjusted Cox models were also run with estimated Monocyte, Bcell, CD4T, CD8T and Natural Killer cell proportions as predictors.

646 Correlation structures for EpiScores, DNAm-based white cell proportions and phenotypic information were assessed using Pearson correlations and pheatmap (Kolde, 2019) (Version 1.0.12) and ggcorrplot packages (Version 0.1.3) (Kassambara, 2019). The psych package (Version 2.0.9) (Revelle, 2020) was used to perform principal components analysis on EpiScores. Figures 1 and 2 were created with BioRender.com.Associations for EpiScores that were related to a minimum of three morbidities were subset from the fully-adjusted Cox proportional hazards results and were visualised using the ggraph package (Version 2.0.5) (Pedersen, 2021). This network representation was used (Figure 5) to highlight protein EpiScores that were connected with multiple morbidities.

\section{Consistency of disease associations between EpiScores and measured proteins}

657

Comparisons were conducted between EpiScore - diabetes associations and diabetes associations with measured proteins using three previous large-scale proteomic studies 660 (Elhadad et al., 2020; Gudmundsdottir et al., 2020; Ngo et al., 2021) In these studies, six cohorts were included (Study 1: KORA n= 993, HUNT n= 940 (Elhadad et al., 2020), Study 2: AGES-Reykjavik n=5,438 and QMDiab n=356 (Gudmundsdottir et al., 2020), Study 3: 663 Framingham Heart Study $n=1,618$ and the Malmo Diet and Cancer Study n=1,221). Study 1 included the KORA dataset, which we use in this study to generate SOMAscan EpiScores. We characterised which SOMAscan-based EpiScore - diabetes associations from our fully- 
adjusted results reflected those observed with measured protein levels. We included basic (nominal $\mathrm{P}<0.05$ ) and fully adjusted results (with either FDR or Bonferroni-corrected $\mathrm{P}<$ 0.05), wherever available, across the lookup cohorts (Supplementary file 1M).

\section{Relationship between EpiScores and COVID-19 outcomes}

Associations between each robust protein EpiScore and subsequent long-COVID or COVID19 hospitalisation were tested in the Generation Scotland population. A binary variable was used for long-COVID based on self-reported COVID-19 duration from the CovidLife study survey 3 questionnaire ( $\mathrm{N}=2,399$ participating individuals) (Fawns-Ritchie et al., 2021). Participants were asked about the total overall time they experienced symptoms in their first/only episode of illness, as well as the whole of their COVID-19 illness and the dataset is correct as of February 2021, when the survey 3 was administered. Of the 9,537 individuals with DNAm that were included in incident disease analyses, 173 indicated that they had COVID-19 and 56 of these individuals reported having long-COVID (> 4 weeks duration of symptoms after infection). Hospitalisation information, derived from the Scottish Morbidity Records (SMR01), was used to obtain COVID-19 hospital admissions using ICD10 codes U07.1 (lab-confirmed COVID-19 diagnosis), and U07.2 (clinically-diagnosed COVID-19). This data linkage identified 268 of the 9,537 individuals that had COVID-19 diagnoses and 29 had been recorded as being hospitalised due to COVID-19. Generalised linear models with either hospitalisation or long-COVID status as binary outcomes were used, with the 109 scaled protein EpiScores as the independent variables. Sex and Age at COVID testing were included as covariates. The latter was defined as the age at positive COVID-19 test or 1st January 2021 if COVID-19 test data were not available. 


\section{Acknowledgements}

691

692 We are grateful to all study participants of KORA, LBC1936, LBC1921 and GS for their 693 invaluable contributions to this study.

694

695 Ethics

696

697 All KORA participants have given written informed consent and the study was approved by 698 the Ethics Committee of the Bavarian Medical Association.

699 All components of GS received ethical approval from the NHS Tayside Committee on 700 Medical Research Ethics (REC Reference Number: 05/S1401/89). GS has also been granted 701 Research Tissue Bank status by the East of Scotland Research Ethics Service (REC 702 Reference Number: 20/ES/0021), providing generic ethical approval for a wide range of uses 703 within medical research.

704 Ethical approval for the LBC1921 and LBC1936 studies was obtained from the Multi-Centre 705 Research Ethics Committee for Scotland (MREC/01/0/56) and the Lothian Research Ethics 706 committee (LREC/1998/4/183; LREC/2003/2/29). In both studies, all participants provided 707 written informed consent. These studies were performed in accordance with the Helsinki 708 declaration. 


\section{Availability of data and materials}

711 Datasets generated in this study are made available in Supplementary file 1; this file includes the protein EpiScore weights for the 109 EpiScores we provide for future studies to use. All results datasets used to create figures are included in Supplementary file 1 and specific locations for these are noted in figure legends and provided here as follows: Figure 2:

\section{files $1 \mathrm{~J}$ and $1 \mathrm{M}$.}

717 All code used in the analyses is available with open access at the following Github repository: https://github.com/DanniGadd/EpiScores-for-protein-levels.

The source datasets from the cohorts that were analysed during the current study are not publicly available due to them containing information that could compromise participant consent and confidentiality. Data can be obtained from the data owners. Instructions for Lothian Birth Cohort data access can be found here:

\section{https://www.lothianbirthcohort.ed.ac.uk/content/collaboration. Dr Simon Cox must be} contacted to obtain a Lothian Birth Cohort 'Data Request Form' by email: simon.cox@ed.ac.uk. Instructions for accessing Generation Scotland data can be found here: https://www.ed.ac.uk/generation-scotland/for-researchers/access; the 'GS Access Request Form' can be downloaded from this site. Completed request forms must be sent to access@generationscotland.org to be approved by the Generation Scotland access committee.

729 Data from the KORA study can be requested from KORA-gen: http://epi.helmholtzmuenchen.de/kora-gen. Requests are submitted online and are subject to approval by the KORA board. 


\section{Competing interests}

R.E.M has received a speaker fee from Illumina and is an advisor to the Epigenetic Clock

Development Foundation. All other authors declare no competing interests.

737

738

739

740

741

742

743

744

745

746

747

748

749

750

751

752

753

754

755

\section{References}

Alatab, S., Sepanlou, S. G., Ikuta, K., Vahedi, H., Bisignano, C., Safiri, S., Sadeghi, A., Nixon, M. R., Abdoli, A., Abolhassani, H., Alipour, V., Almadi, M. A. H., AlmasiHashiani, A., Anushiravani, A., Arabloo, J., Atique, S., Awasthi, A., Badawi, A., Baig, A. A. A., ... Naghavi, M. (2020). The global, regional, and national burden of inflammatory bowel disease in 195 countries and territories, 1990-2017: a systematic analysis for the Global Burden of Disease Study 2017. The Lancet Gastroenterology and Hepatology, 5(1), 17-30. https://doi.org/10.1016/S2468-1253(19)30333-4

Alawieh, A., Farris Langley, E., \& Tomlinson, S. (2018). Targeted complement inhibition salvages stressed neurons and inhibits neuroinflammation after stroke in mice. Science Translational Medicine, 10(441), 6459. https://doi.org/10.1126/scitranslmed.aao6459

Amador, C., Huffman, J., Trochet, H., Campbell, A., Porteous, D., Wilson, J. F., Hastie, N., Vitart, V., Hayward, C., Navarro, P., Haley, C. S., \& Generation Scotland. (2015). Recent genomic heritage in Scotland. BMC Genomics, 16(1), 437. https://doi.org/10.1186/s12864-015-1605-2

Aryee, M. J., Jaffe, A. E., Corrada-Bravo, H., Ladd-Acosta, C., Feinberg, A. P., Hansen, K. D., \& Irizarry, R. A. (2014). Minfi: A flexible and comprehensive Bioconductor package for the analysis of Infinium DNA methylation microarrays. Bioinformatics, 30(10), 
757

758

759

760

761

762

763

764

765

766

767

768

769

770

771

772

773

774

775

776

777

778

779

Bollepalli, S., Korhonen, T., Kaprio, J., Anders, S., \& Ollikainen, M. (2019). EpiSmokEr: A robust classifier to determine smoking status from DNA methylation data. Epigenomics, 11(13), 1469-1486. https://doi.org/10.2217/epi-2019-0206

Brandolini, L., Grannonico, M., Bianchini, G., Colanardi, A., Sebastiani, P., Paladini, A., Piroli, A., Allegretti, M., Varrassi, G., \& Di Loreto, S. (2019). The Novel C5aR Antagonist DF3016A Protects Neurons Against Ischemic Neuroinflammatory Injury. Neurotoxicity Research, 36(1), 163-174. https://doi.org/10.1007/s12640-019-00026-w

Cataldo, A. M., Petanceska, S., Terio, N. B., Peterhoff, C. M., Durham, R., Mercken, M., Mehta, P. D., Buxbaum, J., Haroutunian, V., \& Nixon, R. A. (2004). A $\beta$ localization in abnormal endosomes: Association with earliest $\mathrm{A} \beta$ elevations in $\mathrm{AD}$ and Down syndrome. Neurobiology of Aging, 25(10), 1263-1272. https://doi.org/10.1016/j.neurobiolaging.2004.02.027

Conole, E. L. S., Stevenson, A. J., Green, C., Harris, S. E., Muñoz, S., Valdés-hernández, M. C., Harris, M. A., Bastin, M. E., \& Joanna, M. (2020). An epigenetic proxy of chronic inflammation outperforms serum levels as a biomarker of brain ageing. medRxiv. https://doi.org/https://doi.org/10.1101/2020.10.08.20205245

Deary, I. J., Gow, A. J., Pattie, A., \& Starr, J. M. (2012). Cohort profile: The lothian birth cohorts of 1921 and 1936. International Journal of Epidemiology, 41(6), 1576-1584.

Demichev, V., Tober-Lau, P., Lemke, O., Nazarenko, T., Thibeault, C., Whitwell, H., Röhl, A., Freiwald, A., Szyrwiel, L., Ludwig, D., Correia-Melo, C., Aulakh, S. K., Helbig, E. T., Stubbemann, P., Lippert, L. J., Grüning, N. M., Blyuss, O., Vernardis, S., White, M., ... Kurth, F. (2021). A time-resolved proteomic and prognostic map of COVID-19. Cell Systems, 12(8), 780. https://doi.org/10.1016/J.CELS.2021.05.005 
Elhadad, M. A., Jonasson, C., Huth, C., Wilson, R., Gieger, C., Matias, P., Grallert, H., Graumann, J., Gailus-Durner, V., Rathmann, W., Toerne, C. von, Hauck, S. M., Koenig, W., Sinner, M. F., Oprea, T. I., Suhre, K., Thorand, B., Hveem, K., Peters, A., \& Waldenberger, M. (2020). Deciphering the plasma proteome of type 2 diabetes. Diabetes, 69(12), 2766-2778. https://doi.org/10.2337/db20-0296

Fawns-Ritchie, C., Altschul, D. M., Campbell, A., Huggins, C., Nangle, C., Dawson, R., Edwards, R., Flaig, R., Hartley, L., Levein, C., McCartney, D. L., Bell, D., Douglas, E., Deary, I. J., Hayward, C., Marioni, R. E., McIntosh, A. M., Sudlow, C., \& Porteous, D. J. (2021). CovidLife: a resource to understand mental health, well-being and behaviour during the COVID-19 pandemic in the UK. Wellcome Open Research $20216: 176,6$, 176. https://doi.org/10.12688/wellcomeopenres.16987.1

Friedman, J., Hastie, T., \& Tibshirani, R. (2010). Regularization Paths for Generalized Linear Models via Coordinate Descent. Journal of Statistical Software, 33(1), 1-22.

Fuchsberger, C., Flannick, J., Teslovich, T. M., Mahajan, A., Agarwala, V., Gaulton, K. J., Ma, C., Fontanillas, P., Moutsianas, L., McCarthy, D. J., Rivas, M. A., B Perry, J. R., Sim, X., Blackwell, T. W., Robertson, N. R., William Rayner, N., Cingolani, P., Locke, A. E., Fernandez Tajes, J., ... McCarthy, M. I. (2016). The genetic architecture of type 2 diabetes. Nature Publishing Group, 536, 41-47.

Ganz, P., Heidecker, B., Hveem, K., Jonasson, C., Kato, S., Segal, M. R., Sterling, D. G., \& Williams, S. A. (2016). Development and validation of a protein-based risk score for cardiovascular outcomes among patients with stable coronary heart disease. JAMA, 315(23), 2532-2541. https://doi.org/10.1001/jama.2016.5951

Gold, L., Ayers, D., Bertino, J., Bock, C., Bock, A., Brody, E. N., Carter, J., Dalby, A. B., Eaton, B. E., Fitzwater, T., Flather, D., Forbes, A., Foreman, T., Fowler, C., Gawande, 

B., Goss, M., Gunn, M., Gupta, S., Halladay, D., ... Zichi, D. (2010). Aptamer-based multiplexed proteomic technology for biomarker discovery. PLoS ONE, 5(12), e15004. https://doi.org/10.1371/journal.pone.0015004

GovScot. (2016). Scottish Government. The Scottish Index of Multiple Deprivation (SIMD); 1-20. (2016). Available from: http://www.gov.scot/ Resource/0050/00504809.pdf. Accessed April 2021.

Gudmundsdottir, V., Zaghlool, S. B., Emilsson, V., Aspelund, T., Ilkov, M., Gudmundsson, E. F., Jonsson, S. M., Zilhão, N. R., Lamb, J. R., Suhre, K., Jennings, L. L., \& Gudnason, V. (2020). Circulating protein signatures and causal candidates for type 2 diabetes. Diabetes, 69(8), 1843-1853. https://doi.org/10.2337/db19-1070

Hawksworth, O. A., Li, X. X., Coulthard, L. G., Wolvetang, E. J., \& Woodruff, T. M. (2017). New concepts on the therapeutic control of complement anaphylatoxin receptors. Molecular Immunology, 89, 36-43. https://doi.org/10.1016/j.molimm.2017.05.015

Hay, S. I., Abajobir, A. A., Abate, K. H., Abbafati, C., Abbas, K. M., Abd-Allah, F., Abdulle, A. M., Abebo, T. A., Abera, S. F., Aboyans, V., Abu-Raddad, L. J., Ackerman, I. N., Adedeji, I. A., Adetokunboh, O., Afshin, A., Aggarwal, R., Agrawal, S., Agrawal, A., Kiadaliri, A. A., ... Bryane, C. E. G. (2017). Global, regional, and national disabilityadjusted life-years (DALYs) for 333 diseases and injuries and healthy life expectancy (HALE) for 195 countries and territories, 1990-2016: A systematic analysis for the Global Burden of Disease Study 2016. The Lancet, 390(10100), 1260-1344. https://doi.org/10.1016/S0140-6736(17)32130-X

Hernandez, M. X., Jiang, S., Cole, T. A., Chu, S. H., Fonseca, M. I., Fang, M. J., Hohsfield, L. A., Torres, M. D., Green, K. N., Wetsel, R. A., Mortazavi, A., \& Tenner, A. J. (2017). Prevention of C5aR1 signaling delays microglial inflammatory polarization, favors 
clearance pathways and suppresses cognitive loss. Molecular Neurodegeneration, 12(1), 66. https://doi.org/10.1186/s13024-017-0210-z

830

Hillary, R. F., McCartney, D. L., Harris, S. E., Stevenson, A. J., Seeboth, A., Zhang, Q., Liewald, D. C., Evans, K. L., Ritchie, C. W., Tucker-Drob, E. M., Wray, N. R., McRae, A. F., Visscher, P. M., Deary, I. J., \& Marioni, R. E. (2019). Genome and epigenome wide studies of neurological protein biomarkers in the Lothian Birth Cohort 1936. Nature Communications, 10(1), 3160. https://doi.org/10.1038/s41467-019-11177-x

Hillary, R. F., Stevenson, A. J., McCartney, D. L., Campbell, A., Walker, R. M., Howard, D. M., Ritchie, C. W., Horvath, S., Hayward, C., McIntosh, A. M., Porteous, D. J., Deary, I. J., Evans, K. L., \& Marioni, R. E. (2020). Epigenetic measures of ageing predict the prevalence and incidence of leading causes of death and disease burden. Clin Epigenet, 12(115). https://doi.org/10.1186/s13148-020-00905-6

Hillary, R. F., Trejo-Banos, D., Kousathanas, A., McCartney, D. L., Harris, S. E., Stevenson, A. J., Patxot, M., Ojavee, S. E., Zhang, Q., Liewald, D. C., Ritchie, C. W., Evans, K. L., Tucker-Drob, E. M., Wray, N. R., McRae, A. F., Visscher, P. M., Deary, I. J., Robinson, M. R., \& Marioni, R. E. (2020). Multi-method genome- And epigenome-wide studies of inflammatory protein levels in healthy older adults. Genome Medicine, 12, 60. https://doi.org/10.1186/s13073-020-00754-1

Houseman, E. A., Accomando, W. P., Koestler, D. C., Christensen, B. C., Marsit, C. J., Nelson, H. H., Wiencke, J. K., \& Kelsey, K. T. (2012). DNA methylation arrays as surrogate measures of cell mixture distribution. BMC Bioinformatics, 13, 86. https://doi.org/10.1186/1471-2105-13-86

James, S. L., Abate, D., Abate, K. H., Abay, S. M., Abbafati, C., Abbasi, N., Abbastabar, H., Abd-Allah, F., Abdela, J., Abdelalim, A., Abdollahpour, I., Abdulkader, R. S., Abebe, 

Z., Abera, S. F., Abil, O. Z., Abraha, H. N., Abu-Raddad, L. J., Abu-Rmeileh, N. M. E., Accrombessi, M. M. K., ... Murray, C. J. L. (2018). Global, regional, and national incidence, prevalence, and years lived with disability for 354 Diseases and Injuries for 195 countries and territories, 1990-2017: A systematic analysis for the Global Burden of Disease Study 2017. The Lancet, 392(10159), 1789-1858. https://doi.org/10.1016/S0140-6736(18)32279-7

Jensen, L. J., Kuhn, M., Stark, M., Chaffron, S., Creevey, C., Muller, J., Doerks, T., Julien, P., Roth, A., Simonovic, M., Bork, P., \& von Mering, C. (2009). STRING 8--a global view on proteins and their functional interactions in 630 organisms. Nucleic Acids Research, 37(Database issue). https://doi.org/10.1093/NAR/GKN760

Kamil, B., Anna, F., Anna, S., Sławomir, P., \& Halina, C. (2016). Regulation of sphingomyelin metabolism. Pharmacological Reports, 68(3), 570-581. https://doi.org/10.1016/j.pharep.2015.12.008

Kassam, Z., Belga, S., Roifman, I., Hirota, S., Jijon, H., Kaplan, G. G., Ghosh, S., \& Beck, P. L. (2014). Inflammatory bowel disease cause-specific mortality: A primer for clinicians. Inflammatory Bowel Diseases, 20(12), 2483-2492. https://doi.org/10.1097/MIB.0000000000000173

Kassambara, A. (2019). ggcorrplot: Visualization of a Correlation Matrix using “ggplot2”. $R$ package (Version 0.1.3). https://cran.rproject.org/web/packages/ggcorrplot/ggcorrplot.pdf. Accessed April 2021.

Kim, S.-H., \& Park, M.-J. (2017). Effects of growth hormone on glucose metabolism and insulin resistance in human. Annals of Pediatric Endocrinology \& Metabolism, 22(3), 145-152. https://doi.org/10.6065/apem.2017.22.3.145

Koenig, W., Sund, M., Fröhlich, M., Löwel, H., Hutchinson, W. L., \& Pepys, M. B. (2003). 
Refinement of the Association of Serum C-reactive Protein Concentration and Coronary Heart Disease Risk by Correction for Within-Subject Variation over TimeThe MONICA Augsburg Studies, 1984 and 1987. American Journal of Epidemiology, 158(4), 357-364. https://doi.org/10.1093/AJE/KWG135

Kolde, R. (2019). Pheatmap : Pretty Heatmaps. R package version 1.0.12. https://cran.rproject.org/web/packages/pheatmap/index.html. Accessed April 2021.

Kwak, S. H., \& Park, K. S. (2016). Recent progress in genetic and epigenetic research on type 2 diabetes. Experimental \& Molecular Medicine, 48(3), e220.

Lea, A. J., Vockley, C. M., Johnston, R. A., Del Carpio, C. A., Barreiro, L. B., Reddy, T. E., \& Tung, J. (2018). Genome-wide quantification of the effects of DNA methylation on human gene regulation. ELife, eLife 2018;7:e37513. https://doi.org/10.7554/eLife.37513

Lee, J. K., Jin, H. K., Park, M. H., Kim, B. R., Lee, P. H., Nakauchi, H., Carter, J. E., He, X., Schuchman, E. H., \& Bae, J. S. (2014). Acid sphingomyelinase modulates the autophagic process by controlling lysosomal biogenesis in Alzheimer's disease. Journal of Experimental Medicine, 211(8), 1551-1570. https://doi.org/10.1084/jem.20132451

Liu, Y., Buil, A., Collins, B. C., Gillet, L. C., Blum, L. C., Cheng, L., Vitek, O., Mouritsen, J., Lachance, G., Spector, T. D., Dermitzakis, E. T., \& Aebersold, R. (2015). Quantitative variability of 342 plasma proteins in a human twin population. Molecular Systems Biology, 11(2), 786. https://doi.org/10.15252/MSB.20145728/FORMAT/PDF Lord, J., \& Cruchaga, C. (2014). The epigenetic landscape of Alzheimer's disease. 9, 1921. Lu, A. T., Quach, A., Wilson, J. G., Reiner, A. P., Aviv, A., Raj, K., Hou, L., Baccarelli, A. A., Li, Y., Stewart, J. D., Whitsel, E. A., Assimes, T. L., Ferrucci, L., \& Horvath, S. (2019). DNA methylation GrimAge strongly predicts lifespan and healthspan. Aging, 11(2), 303-327. https://doi.org/10.18632/aging.101684 
Ma, Y., Liu, Y., Zhang, Z., \& Yang, G. Y. (2019). Significance of complement system in ischemic stroke: A comprehensive review. Aging and Disease, 10(2), 429-462. https://doi.org/10.14336/AD.2019.0119

Mantovani, S., Gordon, R., Macmaw, J. K., Pfluger, C. M. M., Henderson, R. D., Noakes, P. G., McCombe, P. A., \& Woodruff, T. M. (2014). Elevation of the terminal complement activation products C5a and C5b-9 in ALS patient blood. Journal of Neuroimmunology, 276(1-2), 213-218. https://doi.org/10.1016/j.jneuroim.2014.09.005

McCartney, D. L., Hillary, R. F., Stevenson, A. J., Ritchie, S. J., Walker, R. M., Zhang, Q., Morris, S. W., Bermingham, M. L., Campbell, A., Murray, A. D., Whalley, H. C., Gale, C. R., Porteous, D. J., Haley, C. S., McRae, A. F., Wray, N. R., Visscher, P. M., McIntosh, A. M., Evans, K. L., ... Marioni, R. E. (2018). Epigenetic prediction of complex traits and death. Genome Biology, 19(1), 136.

McCartney, D. L., Min, J. L., Richmond, R. C., Lu, A. T., Sobczyk, M. K., Davies, G., Broer, L., Guo, X., Jeong, A., Jung, J., Kasela, S., Katrinli, S., Kuo, P. L., Matias-Garcia, P. R., Mishra, P. P., Nygaard, M., Palviainen, T., Patki, A., Raffield, L. M., ... Marioni, R. E. (2020). Genome-wide association studies identify 137 loci for DNA methylation biomarkers of ageing. BioRxiv. https://doi.org/10.1101/2020.06.29.133702

McCartney, D. L., Stevenson, A. J., Hillary, R. F., Walker, R. M., Bermingham, M. L., Morris, S. W., Clarke, T. K., Campbell, A., Murray, A. D., Whalley, H. C., Porteous, D. J., Visscher, P. M., McIntosh, A. M., Evans, K. L., Deary, I. J., \& Marioni, R. E. (2018). Epigenetic signatures of starting and stopping smoking. EBioMedicine, 37, 214-220. https://doi.org/10.1016/j.ebiom.2018.10.051

McCartney, D. L., Stevenson, A. J., Walker, R. M., Gibson, J., Morris, S. W., Campbell, A., Murray, A. D., Whalley, H. C., Porteous, D. J., McIntosh, A. M., Evans, K. L., Deary, I. 
J., \& Marioni, R. E. (2018). Investigating the relationship between DNA methylation age acceleration and risk factors for Alzheimer's disease. Alzheimer's and Dementia: Diagnosis, Assessment and Disease Monitoring, 10, 429-437. https://doi.org/10.1016/j.dadm.2018.05.006

Messner, C. B., Demichev, V., Wendisch, D., Michalick, L., White, M., Freiwald, A., Textoris-Taube, K., Vernardis, S. I., Egger, A. S., Kreidl, M., Ludwig, D., Kilian, C., Agostini, F., Zelezniak, A., Thibeault, C., Pfeiffer, M., Hippenstiel, S., Hocke, A., von Kalle, C., ... Ralser, M. (2020). Ultra-High-Throughput Clinical Proteomics Reveals Classifiers of COVID-19 Infection. Cell Systems, 11(1), 11-24.e4. https://doi.org/10.1016/j.cels.2020.05.012

Min, J. L., Hemani, G., Hannon, E., Dekkers, K. F., Castillo-Fernandez, J., Luijk, R., Carnero-Montoro, E., Lawson, D. J., Burrows, K., Suderman, M., Bretherick, A. D., Richardson, T. G., Klughammer, J., Iotchkova, V., Sharp, G., Khleifat, A. Al, Shatunov, A., Iacoangeli, A., McArdle, W. L., ... Relton, C. L. (2020). Genomic and phenomic insights from an atlas of genetic effects on DNA methylation. MedRxiv. https://doi.org/10.1101/2020.09.01.20180406

Moldoveanu, A. I., Shephard, R. J., \& Shek, P. N. (2000). Exercise elevates plasma levels but not gene expression of IL-1 $\beta$, IL-6, and TNF- $\alpha$ in blood mononuclear cells. Journal of Applied Physiology, 89(4), 1499-1504. https://doi.org/10.1152/JAPPL.2000.89.4.1499/ASSET/IMAGES/LARGE/DG10002090 03.JPEG

Morgan, B. P., \& Harris, C. L. (2015). Complement, a target for therapy in inflammatory and degenerative diseases. Nature Reviews Drug Discovery, 14, 857-877. https://doi.org/10.1038/nrd4657 
MRC-IEU. (2021). The MRC-IEU catalog of epigenome-wide association studies. Avaialable at: http://www.ewascatalog.org. Accessed April 2021.

Navrady, L. B., Wolters, M. K., MacIntyre, D. J., Clarke, T. K., Campbell, A. I., Murray, A. D., Evans, K. L., Seck, J., Haley, C., Milburn, K., Wardlaw, J. M., Porteous, D. J., Deary, I. J., \& McIntosh, A. M. (2018). Cohort profile: Stratifying Resilience and Depression Longitudinally (STRADL): A questionnaire follow-up of Generation Scotland: Scottish Family Health Study (GS: SFHS). International Journal of Epidemiology, 47(1), 13-14g. https://doi.org/10.1093/ije/dyx115

Ngo, D., Benson, M. D., Long, J. Z., Chen, Z. Z., Wang, R., Nath, A. K., Keyes, M. J., Shen, D., Sinha, S., Kuhn, E., Morningstar, J. E., Shi, X., Peterson, B. D., Chan, C., Katz, D. H., Tahir, U. A., Farrell, L. A., Melander, O., Mosley, J. D., ... Gerszten, R. E. (2021). Proteomic profiling reveals biomarkers and pathways in type 2 diabetes risk. JCI Insight, 6(5), e144392. https://doi.org/10.1172/JCI.INSIGHT.144392

NHS England. (2016). Improving Outcomes Through Personalised Medicine. https://www.england.nhs.uk/wp-content/uploads/2016/09/improving-outcomespersonalised-medicine.pdf. Accessed April 2021.

Ort, M., Dingemanse, J., van den Anker, J., \& Kaufmann, P. (2020). Treatment of Rare Inflammatory Kidney Diseases: Drugs Targeting the Terminal Complement Pathway. In Frontiers in Immunology (Vol. 11). Frontiers Media S.A. https://doi.org/10.3389/fimmu.2020.599417

Park, M. H., Jin, H. K., \& Bae, J. sung. (2020). Potential therapeutic target for aging and agerelated neurodegenerative diseases: the role of acid sphingomyelinase. Experimental and Molecular Medicine, 52, 380-389. https://doi.org/10.1038/s12276-020-0399-8

Pedersen, T. L. (2021). ggraph: An Implementation of Grammar of Graphics for Graphs and 
Networks. R package version 2.0.5. https://CRAN.R-project.org/package=ggraph. Accessed April 2021.

Pérez-Pérez, R., García-Santos, E., Ortega-Delgado, F. J., López, J. A., Camafeita, E., Ricart, W., Fernández-Real, J. M., \& Peral, B. (2012). Attenuated metabolism is a hallmark of obesity as revealed by comparative proteomic analysis of human omental adipose tissue. Journal of Proteomics, 75(3), 783-795. https://doi.org/10.1016/j.jprot.2011.09.016

Peters, A., Nawrot, T. S., \& Baccarelli, A. A. (2021). Hallmarks of environmental insults. Cell, 184(6), 1455-1468. https://doi.org/10.1016/j.cell.2021.01.043

Petersen, A. K., Zeilinger, S., Kastenmüller, G., Werner, R. M., Brugger, M., Peters, A., Meisinger, C., Strauch, K., Hengstenberg, C., Pagel, P., Huber, F., Mohney, R. P., Grallert, H., Illig, T., Adamski, J., Waldenberger, M., Gieger, C., \& Suhre, K. (2014). Epigenetics meets metabolomics: An epigenome-wide association study with blood serum metabolic traits. Human Molecular Genetics, 23(2), 534-545. https://doi.org/10.1093/hmg/ddt430

Pietzner, M., Wheeler, E., Carrasco-Zanini, J., Raffler, J., Kerrison, N. D., Oerton, E., Auyeung, V. P. W., Luan, J., Finan, C., Casas, J. P., Ostroff, R., Williams, S. A., Kastenmüller, G., Ralser, M., Gamazon, E. R., Wareham, N. J., Hingorani, A. D., \& Langenberg, C. (2020). Genetic architecture of host proteins involved in SARS-CoV-2 infection. Nature Communications, 11, 6397. https://doi.org/10.1038/s41467-02019996-Z

R. (2020). $R$ Core Team. $R$ : A language and environment for statistical computing. $R$ Foundation for Statistical Computing, Vienna, Austria. URL https://www.R-project.org/. Accessed April 2021.

Revelle, W. (2020). psych: Procedures for Personality and Psychological Research, 

project.org/package=psych. Accessed April 2021.

998

999

1000

1001

1002

1003

1004

1005

1006

1007

1008

1009

1010

1011

1012

1013

1014

1015

1016

1017

1018

1019

Saffari, A., Silver, M. J., Zavattari, P., Moi, L., Columbano, A., Meaburn, E. L., \& Dudbridge, F. (2017). Estimation of a significance threshold for epigenome-wide association studies. 42(1), 22-23.

Safiri, S., Kolahi, A. A., Hoy, D., Smith, E., Bettampadi, D., Mansournia, M. A., AlmasiHashiani, A., Ashrafi-Asgarabad, A., Moradi-Lakeh, M., Qorbani, M., Collins, G., Woolf, A. D., March, L., \& Cross, M. (2019). Global, regional and national burden of rheumatoid arthritis 1990-2017: a systematic analysis of the Global Burden of Disease study 2017. Annals of the Rheumatic Diseases, 78(11), 1463-1471. https://doi.org/10.1136/annrheumdis-2019-215920

Seeboth, A., McCartney, D. L., Wang, Y., Hillary, R. F., Stevenson, A. J., Walker, R. M., Campbell, A., Evans, K. L., McIntosh, A. M., Hägg, S., Deary, I. J., \& Marioni, R. E. (2020). DNA methylation outlier burden, health, and ageing in Generation Scotland and the Lothian Birth Cohorts of 1921 and 1936. Clinical Epigenetics, 12, 49. https://doi.org/10.1186/s13148-020-00838-0

Serban, K. A., Pratte, K. A., \& Bowler, R. P. (2021). Protein biomarkers for COPD outcomes. Chest, ISSN 0012-3692. https://doi.org/10.1016/j.chest.2021.01.004

Shah, S., McRae, A. F., Marioni, R. E., Harris, S. E., Gibson, J., Henders, A. K., Redmond, P., Cox, S. R., Pattie, A., Corley, J., Murphy, L., Martin, N. G., Montgomery, G. W., Starr, J. M., Wray, N. R., Deary, I. J., \& Visscher, P. M. (2014). Genetic and environmental exposures constrain epigenetic drift over the human life course. Genome Research, 24(11), 1725-1733.

Smith, B. H., Campbell, A., Linksted, P., Fitzpatrick, B., Jackson, C., Kerr, S. M., Deary, I. 
J., MacIntyre, D. J., Campbell, H., McGilchrist, M., Hocking, L. J., Wisely, L., Ford, I., Lindsay, R. S., Morton, R., Palmer, C. N. A., Dominiczak, A. F., Porteous, D. J., \& Morris, A. D. (2013). Cohort profile: Generation scotland: Scottish family health study (GS: SFHS). The study, its participants and their potential for genetic research on health and illness. International Journal of Epidemiology, 42(3), 689-700.

Stevenson, A. J., Gadd, D. A., Hillary, R. F., McCartney, D. L., Campbell, A., Walker, R. M., Evans, K. L., Harris, S. E., Spires-Jones, T. L., McRae, A. F., Visscher, P. M., McIntosh, A. M., Deary, I. J., \& Marioni, R. E. (2021). Creating and validating a DNA methylation-based proxy for interleukin-6. Gerontol A Biol Sci Med Sci., 17:glab046. https://doi.org/10.1093/gerona/glab046.

Stevenson, A., McCartney, D., Hillary, R., Campbell, A., Morris, S., Bermingham, M., Walker, R., Evans, K., Boutin, T., Hayward, C., McRae, A., McColl, B., Spires-Jones, T., McIntosh, A., Deary, I., \& Marioni, R. (2020). Characterisation of an inflammationrelated epigenetic score and its association with cognitive ability. Clinical Epigenetics, 12, 113. https://doi.org/10.1101/802009

Suhre, K., Arnold, M., Bhagwat, A. M., Cotton, R. J., Engelke, R., Raffler, J., Sarwath, H., Thareja, G., Wahl, A., Delisle, R. K., Gold, L., Pezer, M., Lauc, G., Selim, M. A. E. D., Mook-Kanamori, D. O., Al-Dous, E. K., Mohamoud, Y. A., Malek, J., Strauch, K., ... Graumann, J. (2017). Connecting genetic risk to disease end points through the human blood plasma proteome. Nature Communications, 8, 14357. https://doi.org/10.1038/ncomms14357

Sun, B. B., Maranville, J. C., Peters, J. E., Stacey, D., Staley, J. R., Blackshaw, J., Burgess, S., Jiang, T., Paige, E., Surendran, P., Oliver-Williams, C., Kamat, M. A., Prins, B. P., Wilcox, S. K., Zimmerman, E. S., Chi, A., Bansal, N., Spain, S. L., Wood, A. M., ... 
Butterworth, A. S. (2018). Genomic atlas of the human plasma proteome. Nature, 558, 73-79. https://doi.org/10.1038/s41586-018-0175-2

Taylor, A. M., Pattie, A., \& Deary, I. J. (2018). Cohort profile update: The Lothian birth cohorts of 1921 and 1936. International Journal of Epidemiology, 47(4), 1042r.

Therneau, T. M. (2020a). A Package for Survival Analysis in $R$. $R$ package version 3.2-7, https://CRAN.R-project.org/package=survival. Accessed April 2021.

Therneau, T. M. (2020b). coxme: Mixed Effects Cox Models. R package version 2.2-16. https://CRAN.R-project.org/package=coxme. Accessed April 2021.

Trejo Banos, D., McCartney, D. L., Patxot, M., Anchieri, L., Battram, T., Christiansen, C., Costeira, R., Walker, R. M., Morris, S. W., Campbell, A., Zhang, Q., Porteous, D. J., McRae, A. F., Wray, N. R., Visscher, P. M., Haley, C. S., Evans, K. L., Deary, I. J., McIntosh, A. M., ... Robinson, M. R. (2020). Bayesian reassessment of the epigenetic architecture of complex traits. Nature Communications, 11 .

Watanabe, K., Taskesen, E., Van Bochoven, A., \& Posthuma, D. (2017). Functional mapping and annotation of genetic associations with FUMA. Nature Communications 2017 8:1, 8(1), 1-11. https://doi.org/10.1038/s41467-017-01261-5

Williamson, E. J., Walker, A. J., Bhaskaran, K., Bacon, S., Bates, C., Morton, C. E., Curtis, H. J., Mehrkar, A., Evans, D., Inglesby, P., Cockburn, J., McDonald, H. I., MacKenna, B., Tomlinson, L., Douglas, I. J., Rentsch, C. T., Mathur, R., Wong, A. Y. S., Grieve, R., ... Goldacre, B. (2020). Factors associated with COVID-19-related death using OpenSAFELY. Nature 2020 584:7821, 584(7821), 430-436.

https://doi.org/10.1038/s41586-020-2521-4

World Health Organization. (2018). Global Health Estimates 2016: Deaths by Cause, Age, Sex, by Country and by Region, 2000-2016. 
Yao, C., Chen, G., Song, C., Keefe, J., Mendelson, M., Huan, T., Sun, B. B., Laser, A., Maranville, J. C., Wu, H., Ho, J. E., Courchesne, P., Lyass, A., Larson, M. G., Gieger, C., Graumann, J., Johnson, A. D., Danesh, J., Runz, H., ... Levy, D. (2018). Genomewide mapping of plasma protein QTLs identifies putatively causal genes and pathways for cardiovascular disease. Nature Communications, 9, 3268.

Zaghlool, S. B., Kühnel, B., Elhadad, M. A., Kader, S., Halama, A., Thareja, G., Engelke, R., Sarwath, H., Al-Dous, E. K., Mohamoud, Y. A., Meitinger, T., Wilson, R., Strauch, K., Peters, A., Mook-Kanamori, D. O., Graumann, J., Malek, J. A., Gieger, C., Waldenberger, M., \& Suhre, K. (2020). Epigenetics meets proteomics in an epigenomewide association study with circulating blood plasma protein traits. Nature Communications, 11(1), 15. https://doi.org/10.1038/s41467-019-13831-w

Zhang, F., Chen, W., Zhu, Z., Zhang, Q., Nabais, M. F., Qi, T., Deary, I. J., Wray, N. R., Visscher, P. M., McRae, A. F., \& Yang, J. (2019). OSCA: A tool for omic-data-based complex trait analysis. Genome Biology, 20(107). https://doi.org/10.1186/s13059-019$1718-\mathrm{Z}$

Zhang, Q., Marioni, R. E., Robinson, M. R., Higham, J., Sproul, D., Wray, N. R., Deary, I. J., McRae, A. F., \& Visscher, P. M. (2018). Genotype effects contribute to variation in longitudinal methylome patterns in older people. Genome Medicine, 10, 75.

Zhang, Y., Wilson, R., Heiss, J., Breitling, L. P., Saum, K. U., Schöttker, B., Holleczek, B., Waldenberger, M., Peters, A., \& Brenner, H. (2017). DNA methylation signatures in peripheral blood strongly predict all-cause mortality. Nature Communications, 6, 10192 .

\section{Additional files}

\section{Supplementary file 1}


(A) Demographic and array information for the cohorts and samples used in the study.

(B) SomaScan panel performance in the STRADL test set.

1094

(C) Performance of Olink protein EpiScores in holdout, STRADL and LBC1921 test sets.

1095

(D) Annotations for the proteins corresponding to the 109 selected EpiScores.

1096

(E) 1E. FUMA canonical pathway GeneSet enrichment results.

1097

(F) Predictor weights for the 109 selected EpiScores.

1098

(G) $\mathrm{CpG}$ feature counts for the 109 selected EpiScores.

1099

(H) Frequency of CpG sites selected for EpiScores with EWAS catalogue annotations to 1100 phenotypic traits.

1101 (I) Basic Cox model results in Generation Scotland.

1102

(J) Fully-adjusted and sensitivity analyses results for Cox models in Generation Scotland.

1103

(K) Schoenfeld residual Cox sensitivity analyses.

1104

1105

1106

1107

1108

1109

1110 morbidities in this study that were used to assign case/control status of participants.

(L) Schoenfeld residual Cox sensitivity analyses split by year of follow-up.

(M) SOMAscan - EpiScore diabetes association lookup against two large-scale plasma protein - diabetes studies.

(N) White blood cell sensitivity analyses.

(O) GrimAge sensitivity analyses.

(P) COVID-19 analyses. (Q-1B1 Primary and secondary health codes for each of the 12

\section{Figure supplements}

1113 Figure 2 - figure supplement 1. Correlation heatmap for protein EpiScore measures in

1114 Generation Scotland. Correlation heatmap for EpiScore measures projected into Generation 
0.05). At the top of the heatmap, an annotation bar is displayed. Olink® proteins are shown

1117 in pale green and Somalogic proteins are shown in purple.

1119 Figure 2 - figure supplement 2. Phenotypic trait and estimated white blood cell

1120 proportion correlations with EpiScores. Heatmap of correlations between the 78 protein

1121 EpiScore measures for Olink ${ }^{\circledR}$ proteins which were associated with incident disease at $\mathrm{P}<$

11220.05 in the fully-adjusted Cox mixed effects proportional hazards models and continuous

1123 phenotypic/lifestyle trait variables and Houseman-estimated white blood cell proportions in

1124 Generation Scotland (total N=9,357). Protein measurements used to train the predictors were

1125 adjusted for age and sex. The maximum sample size available was used for each correlation.

1126 GrimAge: GrimAge acceleration. Units: weekly units of alcohol. EpiSmoker: DNAm-derived

1127 score for smoking. SIMD: Scottish Index of Multiple Deprivation. EA: educational

1128 attainment. Mono: Monocytes. Gran: Granulocytes. NK: Natural Killer cells.

1130 Figure 2 - figure supplement 3. GeneSet enrichment of canonical pathways common to

the genes encoding proteins used to train the 109 robust EpiScores. Genes selected for

1132

pathway enrichment (FDR adjusted $\mathrm{P}<0.05)$ are summarised, with the proportion of

1133

overlapping genes enirched in the gene set also shown. The corresponding data for this figure

1134 can be accessed in full in Supplementary file 1E. 


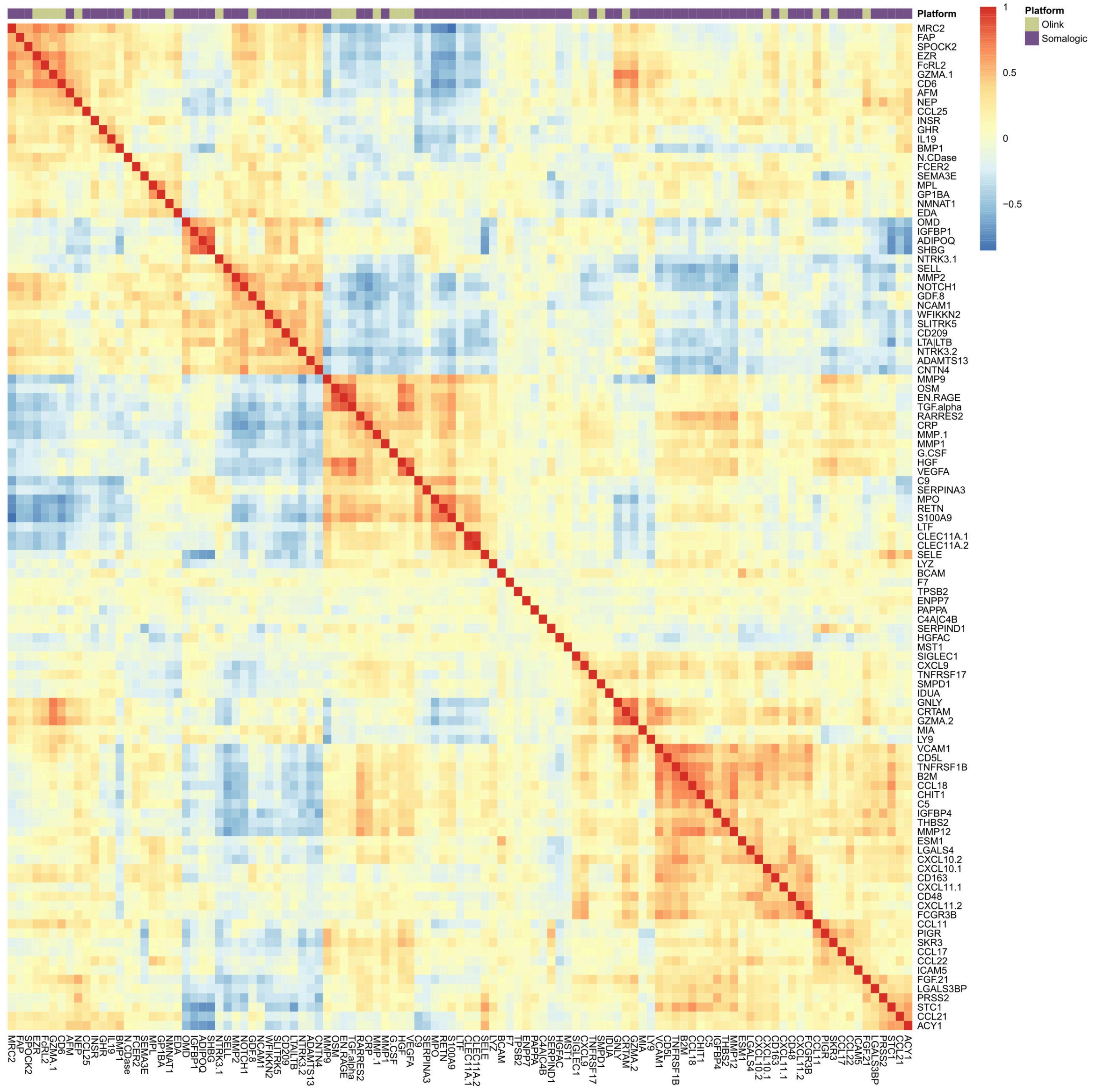




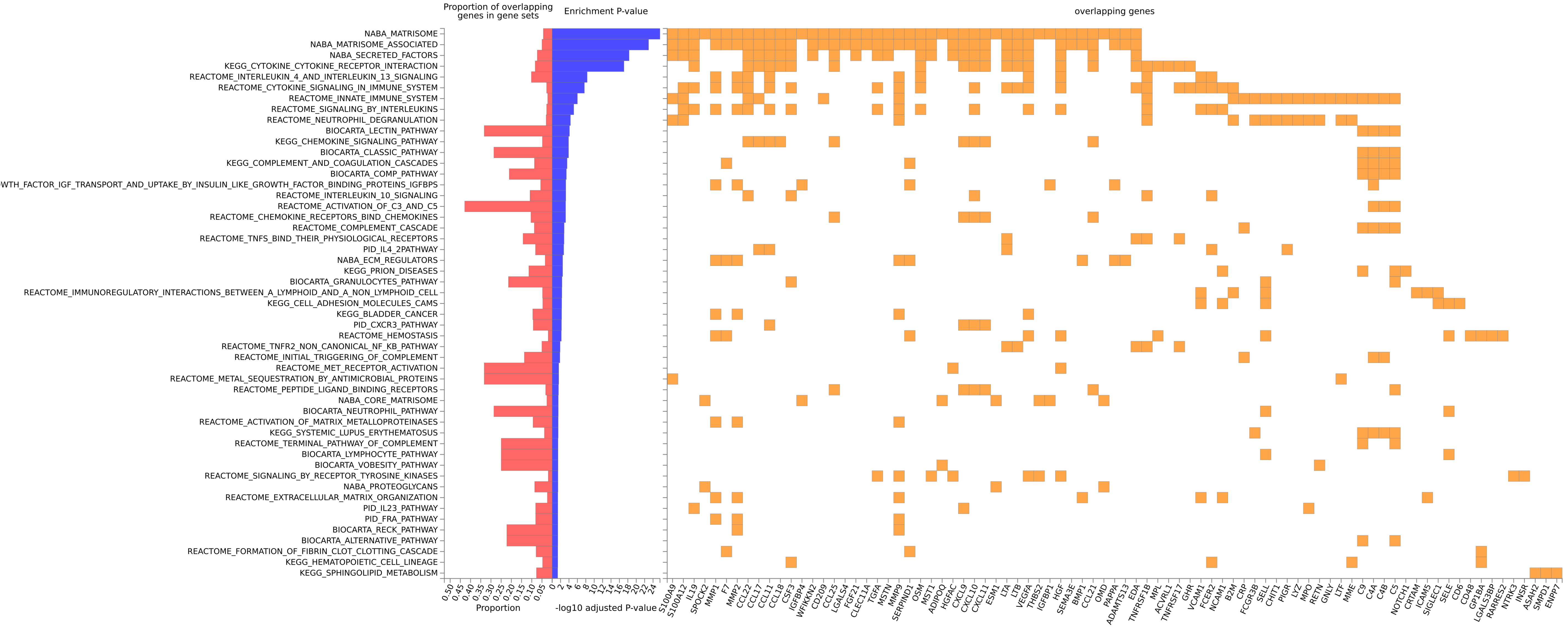

